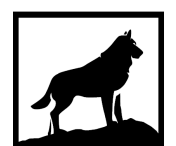

Michigan Technological

1 8 8 5 University
Michigan Technological University Digital Commons @ Michigan Tech

2017

DEVELOPMENT OF AN EVAPORATION SUB-MODEL AND

SIMULATION OF MULTIPLE DROPLET IMPINGEMENT IN VOLUME OF FLUID METHOD

Sathya Prasad Potham

Michigan Technological University, spotham@mtu.edu

Copyright 2017 Sathya Prasad Potham

Recommended Citation

Potham, Sathya Prasad, "DEVELOPMENT OF AN EVAPORATION SUB-MODEL AND SIMULATION OF MULTIPLE DROPLET IMPINGEMENT IN VOLUME OF FLUID METHOD", Open Access Master's Thesis, Michigan Technological University, 2017.

https://doi.org/10.37099/mtu.dc.etdr/536

Follow this and additional works at: https://digitalcommons.mtu.edu/etdr

Part of the Heat Transfer, Combustion Commons 


\title{
DEVELOPMENT OF AN EVAPORATION SUB-MODEL AND SIMULATION OF MULTIPLE DROPLET IMPINGEMENT IN VOLUME OF FLUID METHOD
}

\section{By}

Sathya Prasad Potham

\begin{abstract}
A THESIS
Submitted in partial fulfillment of the requirements for the degree of MASTER OF SCIENCE

In Mechanical Engineering
\end{abstract}

MICHIGAN TECHNOLOGICAL UNIVERSITY

2017

(C) 2017 Sathya Prasad Potham 
This thesis has been approved in partial fulfillment of the requirements for the Degree of MASTER OF SCIENCE in Mechanical Engineering.

Department of Mechanical Engineering - Engineering Mechanics

Thesis Advisor: $\quad$ Dr. Seong-Young Lee

Committee Member: Dr. Youngchul Ra

Committee Member: Dr. Raymond A. Shaw

Department Chair: $\quad$ Dr. William W. Predebon 


\section{To the loving memory of my father.}

\section{To my mother,}

For her unconditional love and unwavering belief in me. 


\section{Table of Contents}

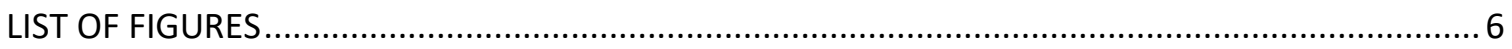

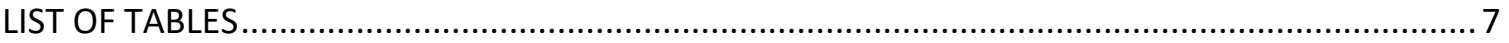

PREFACE

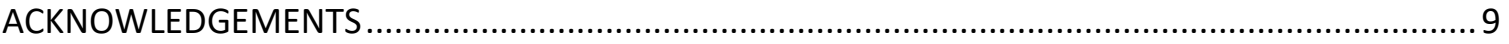

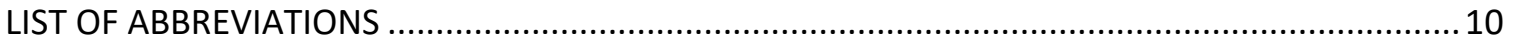

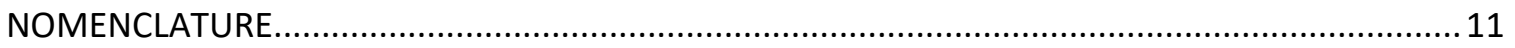

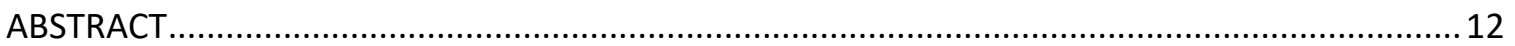

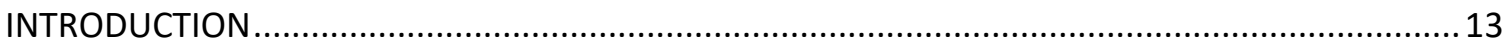

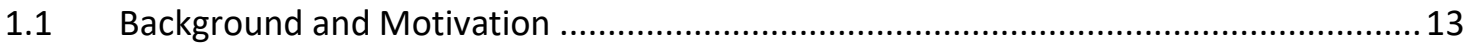

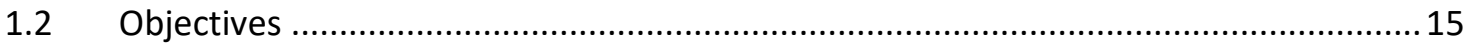

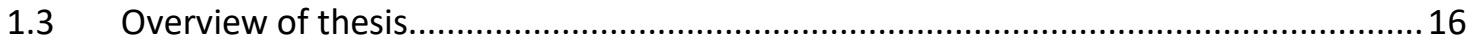

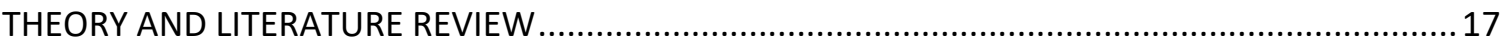

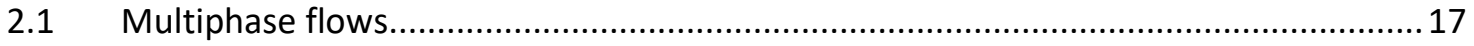

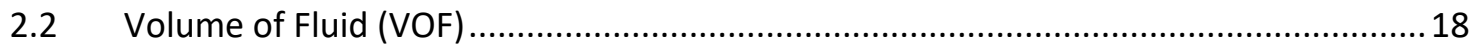

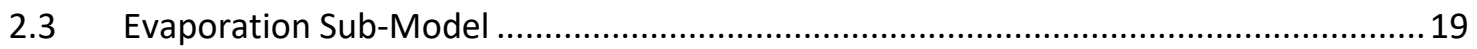

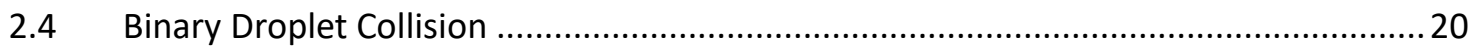

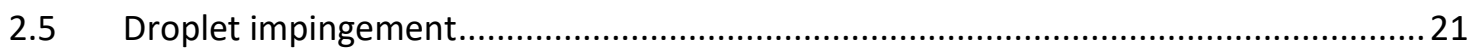

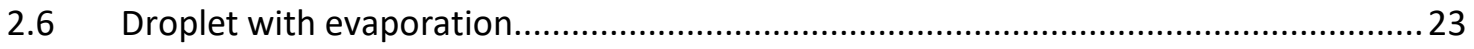

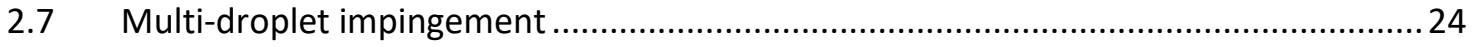

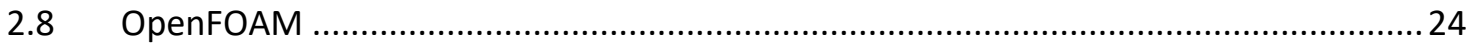

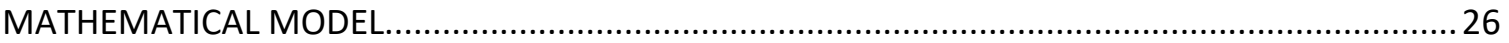

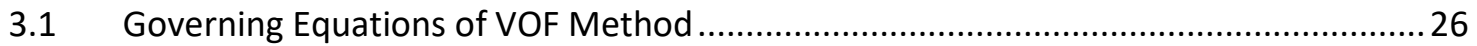

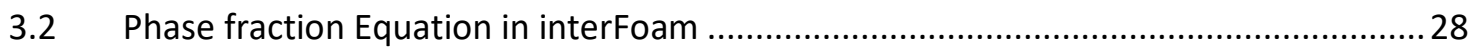

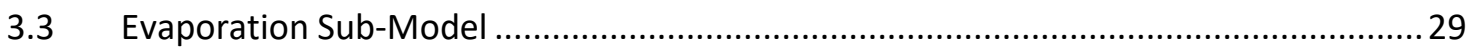

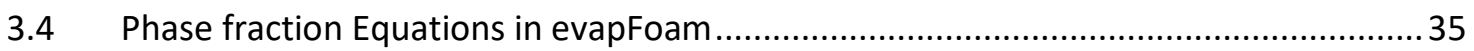

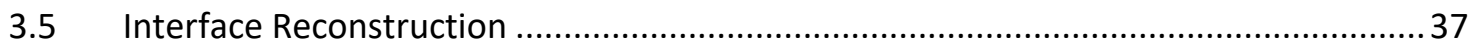

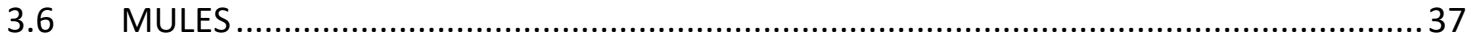

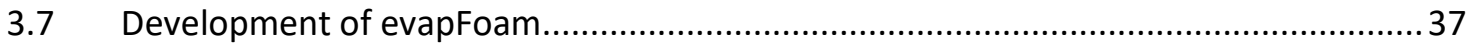

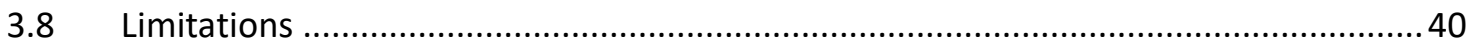

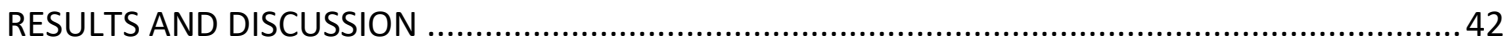




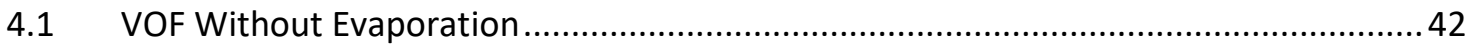

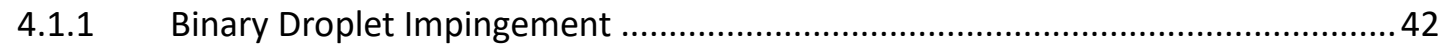

4.1.2 Droplet Impingement On Wet Wall ................................................................... 46

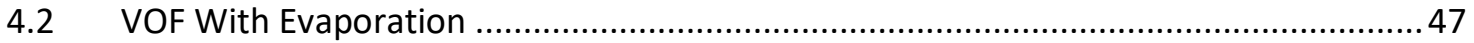

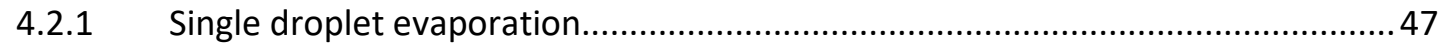

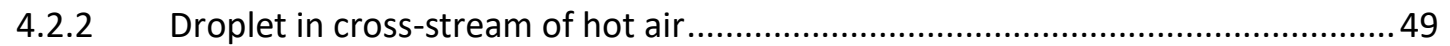

4.3 Multiple Droplet Impingement On Hot Wall .................................................................51

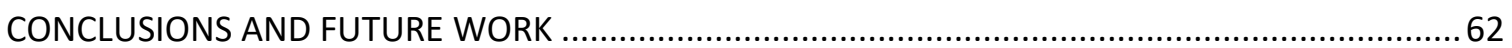

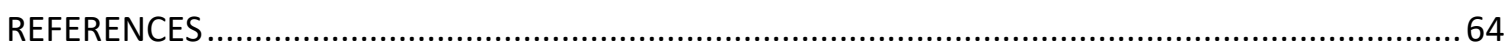

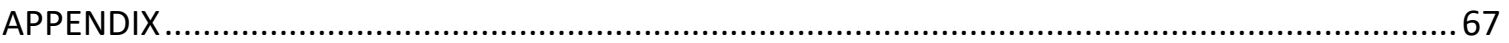




\section{LIST OF FIGURES}

Figure 1: Head-on collision of Water droplets at We-40 from a) Ashgriz and Poo; (1990) b) Simulation.

Figure 2: Time evolution of droplet impingement at impact parameter 0.4 a) Simulation

b)Ashgriz and Poo(1990)... 45

Figure 3: Temporal evolution of water droplet impinging on a wet wall ........................46

Figure 4: Effect of grid resolution on simulation results. ........................................... 48

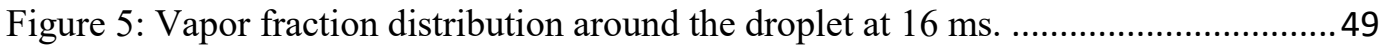

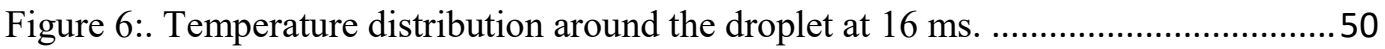

Figure 7: Droplet arrangement of three cases ........................................................... 52

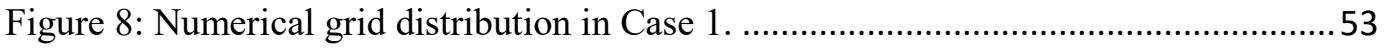

Figure 9: Temporal evolution of liquid and vapor volume fractions..............................56

Figure 10: Droplet lift-off height from the wall......................................................... 58

Figure 11: Temporal variation of average surface temperature ......................................59

Figure 12: Time dependency of liquid mass fraction. .................................................61 


\section{LIST OF TABLES}

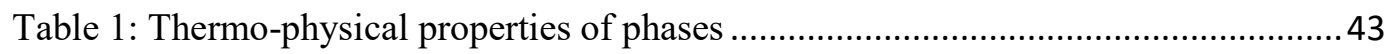

Table 2: Droplet Collisions - Case setup details .............................................................. 43

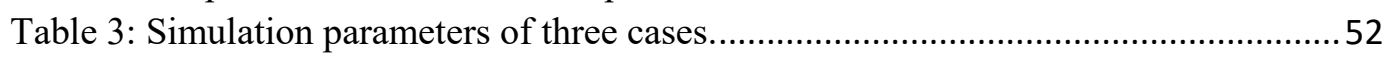




\section{PREFACE}

This thesis contains material previously reviewed and published in scientific journals. Full citation details are provided below

Potham, S., Zhao, L., and Lee, S., "Numerical Study on Evaporation of Spherical Droplets Impinging on the Wall Using Volume of Fluid (VOF) Model," SAE Technical Paper 201701-0852, 2017, doi:10.4271/2017-01-0852.

I developed the numerical model, performed the simulations and wrote the paper. My coauthors contributed in data processing, editing and review of the paper 


\section{ACKNOWLEDGEMENTS}

As the curtain drops on my association with Michigan Tech as a Master's student, I would like to sit back and ponder how things unfolded from the day 1 at Tech and thank people who made this day possible

First and foremost, I would like to thank my advisor Dr. Seong-Young Lee for introducing me to the field of multiphase flows. Dr. Lee had a clear vision of the goals for my research on the very first day he spoke to me about this topic. I thank him from the bottom of my heart for giving me the freedom in choosing the path to achieve those goals and providing me with every essential resource needed. The feedback I received from him after each of our discussions kept me motivated and provided the gentle nudge I sometimes needed.

I thank my committee members Dr. Youngchul Ra and Dr. Raymond Shaw for their valuable feedback on this thesis and their insightful questions which helped me make some valuable corrections.

I also would like to thank my colleagues and officemates Abdul, Emma and Sheldon. Abdul was the first student I came to know from Dr. Lee's group and he gave me a glimpse of Dr. Lee's research work the first time I met him, which eventually helped me in deciding to join Dr. Lee's research group. Emma deserves a special mention for the patience she had in constantly making sure of my pace in my research. I thank her for her valuable opinions on various ideas I had throughout my research. I thank Sheldon for teaching me a lot about CV Lab which helped me to make some contributions to few other projects of Dr. Lee. Sheldon's perseverance and focus on his research in the projects I collaborated with him made me set some new goals for myself. I also would like to thank my roommates and friends Venkatesh, Ekanth, Hrishikesh for many light-hearted moments and nostalgic stories we shared over the dining table. Lastly, I thank my mother Rajeswari and my sister Lakshmi for their love and support throughout my life. 


\section{LIST OF ABBREVIATIONS}

$\begin{array}{ll}\text { CFD } & \text { Computational Fluid Dynamics } \\ \text { CSF } & \text { Continuum Surface Force } \\ \text { FVM } & \text { Finite Volume Method } \\ \text { IC } & \text { Internal Combusion } \\ \text { KH-RT } & \text { Kelvin Helmholtz Rayleigh Taylor } \\ \text { LHS } & \text { Left Hand Side } \\ \text { MAC } & \text { Marker And Cell } \\ \text { MULES } & \text { Multi-dimensional Limiter with Explicit Solution } \\ \text { PLIC } & \text { Piece-wise Linear Interface Calculation } \\ \text { RHS } & \text { Right Hand Side } \\ \text { OpenFOAM } & \text { Open Source Field Operation And Manipulation } \\ \text { UDF } & \text { User Defined Function } \\ \text { VOF } & \text { Volume Of Fluid }\end{array}$




\section{NOMENCLATURE}

c Specific heat

D Diffusivity

g Gas phase

h Latent heat

I Liquid phase

m Mass

n Normal

p Pressure

sat Saturation

T Temperature

U Velocity vector

v Vapor phase

Y Mass fraction

a Phase fraction

$\lambda$ Thermal conductivity

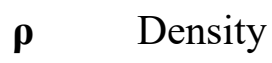

$\boldsymbol{\mu} \quad$ Dynamic viscosity

v Kinematic viscosity

б Surface tension coefficient

к Curvature 


\section{ABSTRACT}

Droplet collision and impingement on a substrate are widely observed phenomenon in many applications like spray injection of Internal Combustion Engines, spray cooling, spray painting and atomizers used in propulsion applications. Existing Lagrangian models do not provide a comprehensive picture of the outcome of these events and may involve model constants requiring experimental data for validation. Physics based models like Volume of Fluid (VOF) method involve no parametric tuning and are more accurate. The aim of this thesis is to extend the basic VOF method with an evaporation sub-model and implement in an open source Computational Fluid Dynamics (CFD) software, OpenFOAM. The new model is applied to numerically study the evaporation of spherical n-heptane droplets impinging on a hot wall at atmospheric pressure and a temperature above the Leidenfrost temperature. An additional vapor phase is introduced apart from the liquid and gas phases to understand the mixing and diffusion of vapor and gas phases. The evaporation model is validated quantitatively and qualitatively with fundamental problems having analytical solutions and published results. The effect of droplet number and arrangement on evaporation is studied by three cases with one (Case 1), two (Case 2) and four (Case 3) droplets impinging on hot wall in film boiling regime at a fixed temperature of wall and a constant non-dimensional distance between droplets. Droplet lift and spread, surface temperature, heat transfer, and evaporation rate are examined. It was observed that more liquid mass evaporated in Case 1 compared to the other cases. Droplet levitation begins early in Case 1 and very high levitation observed was partially due to contraction

of its shape from elongated to a more circular form. Average surface temperature was also considerably reduced in Case 1 due to high droplet levitation. 


\section{CHAPTER 1 INTRODUCTION}

\subsection{Background and Motivation}

Liquid sprays with droplets impinging on a surface, droplet-droplet and jet-jet collisions are widely observed phenomena in many natural and technological applications $[1,2]$. Water droplet collisions formed bulk of early research due to their application in meteorological field [3, 4]. Later studies focused on hydrocarbon droplet collisions in internal combustion engines and gas turbine environment, jet to jet collisions in atomizers used in propulsion and material processing and jet to surface collisions in electronic cooling and spray painting applications $[5,6]$. The outcome of the impingement process ranges from rebound, stick, splash to breakup and is dependent on inertial, surface tension and viscous forces of the impinging spray or droplets [7]. It is a complex phenomenon depending on many non-dimensional flow parameters like Reynolds number and Weber number. In many applications, spray and droplet impingement is often accompanied with a phase change phenomenon. The liquid, vapor and the surrounding air form a multiphase system. The liquid boundary is defined by an interface and separates it from the continuous phase consisting of vapor and air. Multiphase flows with interfacial phase change play a dominant role in many industrial applications like cavitating pumps, boilers, condensers, liquid spray cooling and internal combustion engines.

In internal combustion engines, evaporation of the liquid fuel to vapor phase is one of the critical requirements for combustion of fuel. This phase change is driven by the temperature and mass fraction gradients between the liquid and vapor phase. A lot of advancements have been introduced in fuel injection technology to enhance atomization and evaporation of fuel. It is desirable to reduce wall wetting either by reducing liquid spray penetration or by ensuring liquid spray rebounds from the wall. In spray cooling applications, it is important to achieve sticking of spray or droplets onto the wall and obtain maximum heat flux transfer from wall to liquid with minimum evaporation [8]. Multiple 
liquid inter jet impingement is used to increase the atomization efficiency leading to the formation of ligaments and small droplets in atomizers used in rocket injectors. In other applications like spray coating, phase change is not desired and liquid spray is expected to form a film on the wall. Some applications like refrigeration cycles involve multiphase flows along with interfacial phase change but doesn't involve spray impingement.

In all these applications, the fundamental phenomena are droplet - droplet, droplet - wall interactions, formation of thin films, heat and mass transfer, bubble generation and interaction between the phases. It is important to understand these phenomena to improve their design and operational efficiencies. Though experimental research paved the way for initial stages of development, it posed difficulties in studying certain conditions like microgravity, small droplets and high temperatures. Experimental studies also involve significant costs and time. This difficulty can be overcome using comprehensive mathematical models to numerically simulate the physical process to determine phase composition and amount of energy transfer at various time and length scales. CFD models provide the advantage of cost and time saving when employed for studying the effects of process parameters wherein multiple simulations would be needed. The highly detailed results of these parameters are important for product design and development.

Currently, the most widely used models are computationally simple, involve a lot of parameters and are developed for specific flow conditions. Their applicability to different flow scenarios requires parameter tuning and many not result in best of solutions for various cases. Traditional collision models like O'Rourke models only coalescence and bouncing outcomes and is found to be inaccurate in modeling a wide range of collision outcomes in droplet-droplet and droplet-wall impingement conditions [9]. Droplet vaporization models like Frossling, Chiang correlations assume a spherical shape and are not accurate for irregular shaped ligaments formed generally due to droplet-droplet and droplet-wall interactions. Spray breakup models like KH-RT assume blobs in the place of liquid core and are inaccurate in modeling jet-jet interactions. Hence, physics based models are necessary to improve the accuracy of simulations and to be used as a substitute for 
experiments. They can also be used to validate the simpler parametric models for different applications. Recent advancements in computational technology makes the use of such high-fidelity models more desirable.

\subsection{Objectives}

Binary droplet collision and droplet impingement of water droplets are widely researched in isothermal conditions. Most of those studies focused on understanding the effect of process parameters on the collision outcomes and preparing a collision regime map. Phase change models presented in various papers discussed the implementation of evaporation sub-model in either VOF or Level set method. Mathematical models for implementation of evaporation in VOF are mostly similar irrespective of the CFD software code used for their implementation. Most of the research in this field dealt with only liquid and surrounding gas phases and neglecting the vapor phase. It is important to differentiate the vapor and surrounding gas phases in many applications like IC Engines, where the mixing of fuel in vapor phase with surrounding air is critical for quality of combustion. A few papers discussed binary droplet collision and droplet impingement on hot walls with the evaporation model in VOF. It is quite uncommon to have isolated droplet-droplet or droplet-wall interactions in practical applications. Every droplet interaction will be surrounded by similar interactions which might affect the outcome of each other. Very little research has been done in understanding the interaction effects in multiple droplet impingement. There is a need to bridge the gap in understanding of jet impingement and droplet impingement in non-isothermal evaporating conditions.

This thesis aims to take few steps in improving understanding of multiple droplet impingement phenomenon. Few important objectives of this study are:

* To demonstrate the effectiveness of VOF method implemented in OpenFOAM to simulate binary droplet impingement and droplet impingement on a wall.

* To develop and implement an evaporation model in OpenFOAM as an extension to the already available multi-phase flow solvers without phase change. 
- To study spatially distributed multi-droplet impingement on hot walls with focus on droplet interaction effect on evaporation rate, droplet spread and levitation.

\subsection{Overview of thesis}

Chapter 2 of this thesis discusses in detail the theory of droplet collisions, droplet impingement, multiphase flows along with VOF method. It also introduces OpenFOAM code and highlights the advantages in choosing it. This chapter also gives a review of the literature in chronological order contributing to each of these topics.

Chapter 3 presents the governing equations of VOF method with and without the evaporation model. It also discusses the modified form of the equations suitable for implementation in OpenFOAM. This chapter concludes with the description of sequence of steps the CFD code follows.

Chapter 4 details the results of the project in three parts. The first part focuses on the results obtained with interFoam solver in OpenFOAM. Second part presents the results obtained with the evaporation solver developed in this project and compares the results with the published literature to validate the solver. In the third part, multiple droplet impingement is discussed. Detailed description of the cases will be followed by the discussion on mechanism of droplet interaction and concluding with the discussion on trends of macro indicators like liquid mass fraction percentage, average surface temperature and droplet levitation.

Chapter 5 summarizes the findings of the thesis with important conclusions. It also recommends few steps to continue this work in future to obtain a more comprehensive understanding of the topic. 


\section{CHAPTER 2 THEORY AND LITERATURE REVIEW}

\subsection{Multiphase flows}

Multiphase flows are flows with more than one component or phase and are generally separated by an interface. Each of these phases has a unique set of transport properties, occupies certain volume and moves with certain velocity. There could be an exchange of momentum, heat and mass between the phases. Two phase flows are widely studied due to their presence in many natural and industrial phenomena. They include a broad spectrum of flows like solid-liquid flows or liquid-liquid flows or liquid-gas flows. Each phase could be either continuous or dispersed. In general, gas or liquid phases are continuous and liquid or solid phases are discrete.

Multiphase flows can be studied either experimentally or theoretically or computationally. Experimental setup of various flow scenarios may not be feasible due to significant cost and time requirements or measurement difficulties at the required time and length scales. So theoretical and computational models present an alternative for exploring multiphase flows. With the improvement of computational infrastructure over recent years, detailed numerical simulations of various physical phenomena can now be performed in reasonable amount of time. Based on the numerical treatment of each phase, multiphase flow models can be classified as Eulerian-Eulerian models, Eulerian-Lagrangian models and Eulerian models.

Eulerian-Eulerian models use a two fluid approach and both the fluids/phases are treated as continuous and a separate set of Navier-Stokes equations is solved for each of the phases. It is suitable for flows with similar volume fractions for each phase as it attempts to solve each phase at every point in space. This makes the Eulerian-Eulerian models computationally expensive. The interaction among the phases is vice versa and is modeled. In Eulerian models, all the phases are treated together as a single phase based on volume averaging of each phase. Only one set of governing equations are solved for all the phases 
together. This makes Eulerian method computationally simpler compared to EulerianEulerian methods. Volume of Fluid method and Level set method are two of the most frequently used Eulerian models. In Eulerian-Lagrangian models, dispersed phase is solved in Lagrangian and the continuum phase in Eulerian framework. The dispersed phase is expected to occupy negligible volume and hence only the effects of continuum phase on Lagrangian phase are modeled and the reverse is neglected. Physical processes like collision, drag, atomization and breakup of droplets are modeled. Hence, this method does not require a very fine mesh and is computationally simple. Though, this makes the Lagrangian models to be widely used, it is to be noted that these models are to be tuned to obtain better results by comparing with the experimental results. This makes their applicability limited.

Irrespective of the method, modeling of multiphase flows has some hurdles owing to the inherent complex nature of the flows. The interfaces separating different phases in a multiphase flow introduce discontinuities in the domain. These discontinuities in physical properties present challenges in numerical modeling. Modeling of physical processes across the interface like heat and mass transfer is also difficult since the interfaces are continuously moving and their position and shape are not known at the start.

\subsection{Volume of Fluid (VOF)}

VOF belongs to the class of Eulerian method of solving multiphase flows. It is opted in this study as it is physics based and requires no modeling or parameter. A finite difference technique, Marker and Cell (MAC) method was developed by Francis Harlow and his team at Las Alamos Laboratories [10]. It served as a precursor for the development of VOF method in later years. VOF method is a numerical technique developed by Hirt and Nichols for tracking the free surface in two phase flows [11]. It was successfully applied to simulate various physical problems including sprays and droplet impingement. In VOF method, a volume fraction variable describes the volume of each phase in a cell and is transported across the grid. One momentum equation is solved for both the phases. An interface reconstruction method is used along with the volume fraction solution to accurately 
describe the interface. Geometric reconstruction methods like Piece-wise Linear Interface Calculation (PLIC) are more accurate than algebraic interface reconstruction. VOF method is widely implemented in latest computer codes along with Piece-wise Linear Interface Calculation (PLIC) scheme for interface reconstruction. Level set method is another Eulerian method and is an alternative to VOF method. In level set method, a level set function is transported across the domain with its value being zero at the interface. Level set method results in a more accurate description of the interface compared to the Volume of Fluid method. But VOF method is more preferred in many applications as it results in better mass conservation compared to the Level Set method.

\subsection{Evaporation Sub-Model}

VOF method was extended to model phase change in the last decade. Welch and Wilson used VOF method for modeling phase change in liquid flows as it exhibits the mass conservation in flows without phase change [12]. Mass transfer during the phase change is driven by the gradient of heat flux vector. The model was applied to study horizontal film boiling. Interface was assumed to be at saturation temperature in this model. Hardt and Wondra considered the superheat of interface temperature in modeling the evaporation source term [13]. They used an inhomogeneous Helmholtz equation to adjust the location of the vaporization source to accurately calculate the microscale evaporation. Volume of Fluid method with PLIC was used for describing the interface motion. Zhang presented a numerical model to simulate vaporization of $n$-heptane droplet in forced convection environment [14]. He compared the results of simulations at zero gravity conditions with the experiments of Nomura et al. under micro-gravity conditions [15]. He found that droplet diameter decreases as per the D2-law only at lower ambient pressures and droplet lifetime decreases with increase in ambient temperature. Schlottke and Weigand developed a model for direct numerical simulation of evaporation in an incompressible flow using FS3D, an in-house code [16]. A VOF based method was used to model phase change in the presence of three phases. The model was applied for three dimensional simulation of evaporating droplets. The interface is reconstructed using PLIC and gaseous and liquid 
phase velocities are calculated separately for accurate prediction of the interface movement. Kunkelmann and Stephan implemented and validated the model proposed by Hardt and Wondra in OpenFOAM $[13,17]$. They also extended the model to include contact line evaporation. Sun et al. used user-developed functions (UDFs) in Fluent to model phase change with evaporation and condensation in Volume of Fluid method [18]. A geometric reconstruction scheme PLIC was used to simulate interface motion. The model was verified to produce grid independent and accurate results.

\subsection{Binary Droplet Collision}

Droplet collision is a frequent event in dense region of sprays in fuel injections in IC Engines [19]. Ashgriz and Givi conducted experimental studies on binary droplet collisions of burning and non-burning fuel droplets to determine the influence of internal combustion engine like high temperature environment on droplet collisions [20]. Later Asgriz and Poo carried out experiments with water droplets of different diameter ratios and weber numbers to determine the collision regimes [21]. The behavior of droplet collision with another droplet or a wall is mainly dependent on flow properties like Weber number, Reynolds number, droplet diameter ratio, impact parameter and surroundings including temperature, density of surrounding gas.

$$
\begin{aligned}
& W e=\frac{\rho * U^{2} * D}{\sigma} \\
& R e=\frac{\rho * U * D}{\mu}
\end{aligned}
$$

Impact parameter is the ratio of the distance between center of one droplet to the relative velocity vector placed on center of another droplet to the sum of radii of the droplets. It characterizes the eccentricity between droplets. In binary droplet head-on collisions, bouncing takes place at lower Weber numbers. The collision outcome changes to coalescence and reflexive separation as Weber number increases. Stretching separation is observed in off-axis collisions. Jiang et al. conducted experiments on hydrocarbon droplet collisions and observed that the collision outcomes are different compared to water droplet 
collisions for same conditions [22]. Rieber and Frohn used VOF method with the second order interface reconstruction [23]. The results of the regime transition from coalescence to separation were compared to the experiment. Nobari et al. solved two separate sets of Navier Stokes equations for droplets and surroundings to model head on collisions [24]. The simulations focus on rupture of thin film between droplets during coalescence. Qian and Law presented time resolved photographs of various collision regimes of water and hydrocarbon droplets in different gases and pressures [5]. Pan and Suga used level set method to simulate binary droplet collisions of water and hydrocarbon droplets [25]. The simulation results were compared to experimental results in every collision regime. It was concluded that end pinching was the main reason for satellite droplet formation in head on collisions whereas twisting and stretching dominate in off-axis collisions. Nikolopoulas et al. used VOF method with adaptive local grid refinement technique to simulate droplet collision. They used two VOF indicator functions to distinguish between droplets [26]. Li and Fritsching studied binary droplet collisions using VOF method along with ghost cell method [27]. Droplet bouncing could be simulated with the help of ghost cell method. Saroka et al. performed numerical simulations of water, mercury and tetradecane droplets in inert environment using VOF method [28]. The simulation results were used to assess some assumptions used in models about the collision outcomes.

\subsection{Droplet impingement}

Though experimental studies on droplet impingement were begun in early $20^{\text {th }}$ century, numerical studies began only in later half of the century. Harlow and Shannon were the first to numerically study the splashing of the droplet impinging on solid and liquid surfaces [29]. They used the MAC method developed earlier for this study. The outcome of droplet impingement is dependent on Weber number, Reynolds number along with temperature, surface roughness of the wall. In droplet wall interactions, droplet bounces off the wall at low Weber number and Reynolds number. At higher weber number, droplet deposition takes place. Droplet spreads onto the wall without breaking or producing child droplets. When the weber number is much higher, droplets breaks on impact. The physics of droplet- 
wall interaction is different in the case with evaporation. The evaporation of the droplet when the wall temperature is below the saturation temperature of the droplet is driven by the vapor diffusion. In case of wall temperature above the saturation temperature, heat transfer from the wall to the droplet is the prime driving force for evaporation of the droplet. Evaporation of the droplet enters nucleation regime when the wall temperature increases beyond saturation temperature. In this regime, vapor bubbles are formed as the droplet approaches the wall. The number and lifetime of the vapor bubbles increases with the wall temperature. When the wall temperature increases above Leidenfrost temperature, a thin vapor film is formed separating the droplet and the wall. Droplet levitates and doesn't come in contact with the wall. The thermal conductivity of the vapor is less and hence the heat flux to the droplet decreases. Foote studied liquid droplet behavior using an extension of MAC method to include surface tension effects [30]. Madejski uses overall energy balance of the droplet for solving droplet spreading and solidification [31]. This method doesn't involve solving Navier Stokes equations, but solves velocity profile satisfying continuity equation. Trapaga and Szekely used a VOF method based commercial code Flow-3D to study droplet spreading upon isothermal impact on a solid substrate [32]. Fukai et al. solved numerically a set of finite element equations built on a theoretical model of droplet deformation during its impact on a flat wall [33]. The focus of the study was on understanding the effect of droplet diameter, velocity, material properties and surface tension. The results demonstrated the spread and recoil motion of the droplet along with the mass accumulation on the splat periphery. Initial VOF method was not very accurate in predicting the interface. Rein presented thermal and physical aspects of droplet interactions with solid and liquid surfaces [4]. He discussed numerical models suitable for droplet impingement studies including VOF and level set methods. Bussmann et al. developed a mathematical model based on RIPPLE to simulate droplet impact on asymmetric surfaces [34]. This model uses contact angles as function of contact velocities. Kamnis and Gu studied the droplet impingement dynamics to better understand the thermal spray process [35]. They studied the effect of thermal contact stress on spreading, solidification and air entrapment of droplets impinging on the substrate. They used VOF 
method with geometric reconstruction of interface available in Fluent. The model was applied to simulate the impingement of tin droplets on a steel substrate and the results are compared to the experiments. Nikolopoulos et al. simulated droplets impinging on a wall film using VOF method [36]. They used an adaptive local grid refinement for refining interface separating liquid and gaseous phases. The effect of weber number on size and number of secondary droplets formed after droplet impingement is studied. Mesh dependency studies showed that the droplet hydrodynamics is mesh dependent at higher weber numbers.

\subsection{Droplet with evaporation}

Nguyen and Avedisian studied numerically the film evaporation of liquid droplets on a hot surface [37]. They studied the isothermal and adiabatic surface cases. The time for evaporation of a droplet decreases with increase in temperature of isothermal wall or increase of ambient temperature in case with the adiabatic wall. Pasandideh et al. used a modified SOLA-VOF model to model tin droplet impingement on a hot substrate and study the heat transfer in the droplet and the substrate [38]. Harvie et al. modeled deformation of the droplet hitting the substrate and also the fluid flow within the viscous sub layer using VOF method coupled with a one dimensional algorithm [39]. Nikolopoulas et al. investigated evaporation of $\mathrm{n}$-heptane and water droplets upon impingement on hot wall [40]. Volume of Fluid (VOF) method was used for simulating flow hydrodynamics and vapor phase, mass transfer during phase change was calculated using an evaporation model. The effect of temperature of the wall is explored with cases below and above Leidenfrost temperature. Mahulkar et al. used VOF method with geometric reconstruction of the interface to obtain the regime maps of hydrocarbon droplet impingement on a heated wall [41]. Impingement regimes of splash, stick, rebound and breakup are predicted with CFD simulations for single and multi-component liquids with different diameters. The results are used to compare and validate the correlations for estimating post-impact behavior of droplet-wall interaction developed using energy analysis. 


\subsection{Multi-droplet impingement}

Though single droplet impingement is a fundamental aspect of spray impingement and is widely researched, the results of these studies cannot be directly extrapolated to gain accurate understanding of spray impingement. Interactive effects between droplets, droplet and film, splash crowns and droplet - droplet interaction form other important facets of spray impingement study. Soriano et al. presented an experimental study on cooling effects of single and multiple droplet impingement [42]. Droplet frequency, flow rate and fluid temperature were varied to observe the effect on surface temperature. It was found that multiple droplets and higher flow rate resulted in higher heat flux. Lewis et al. investigated the differences in cooling behavior of droplet train and jet impingement on hot and wetted wall using the VOF method in OpenFOAM [43]. It was found that jets with fully developed velocity profile are more effective in heat transfer compared to the jets with uniform velocity and droplet train.

\subsection{OpenFOAM}

OpenFOAM (Open source Field Operation and Manipulation) is free, open source CFD software package developed by contributors led by Henry Weller, OpenCFD Ltd and the OpenFOAM foundation [44, 45]. It is released under GNU General Public License version 3. It's a C++ toolbox for solving a wide variety of problems like flows, chemical reactions, heat transfer, turbulence, solid mechanics, acoustics and electromagnetism. It is based on Finite Volume Method (FVM). Henry Weller chose $\mathrm{C}++$ instead of Fortran for its modularity and Object Oriented features. OpenFOAM, in its basic version, comes with source code and pre-compiled binaries for sample solvers and utilities. Users can build custom objects, solvers, utilities without effecting the existing code. Third party package OpenMPI is used to provide parallel functionality to OpenFOAM. The main advantage of OpenFOAM comes from the fact that it is an open source software with transparent source code and is scalable for a large number of processors on multi-clustered machines. This 
helps in seamless transfer of knowledge between user communities in academia and industry.

OpenFOAM has in-built solvers for multiphase flows. Some of the solvers are based on mixture model of Volume of Fluid method and some are on Euler-Euler two fluid model. Volume of Fluid method in OpenFOAM uses an algebraic reconstruction method instead of a geometric reconstruction method like PLIC. The algebraic method based on interface compression is faster than PLIC, but it's accuracy depends on the mesh refinement. The phase change solver developed in the current study is built based on interPhaseChangeFoam, a VOF solver with cavitation sub-model in OpenFOAM. 


\section{CHAPTER 3 \\ MATHEMATICAL MODEL}

\subsection{Governing Equations of VOF Method}

The conservation laws of mass and momentum are used to describe the fluid motion of isothermal, single phase flows. Multiphase flows involving two or more phases require additional equations to describe each of the additional phases and the relation between phase properties. These additional equations are transport equations of volume fraction variables and are solved to capture the interface. They are solved simultaneously with the conservation equations of mass and momentum. The conservation of mass is expressed as continuity equation. The momentum equation is obtained by balancing the total forces acting on a fluid element with gravity forces, viscous forces, surface tension and body forces.

$$
\begin{gathered}
\frac{\partial \rho}{\partial t}+\boldsymbol{\nabla} \cdot(\rho \boldsymbol{U})=0 \\
\frac{\partial(\rho \boldsymbol{U})}{\partial t}+\boldsymbol{\nabla} \cdot(\rho \boldsymbol{U} \otimes \boldsymbol{U})=-\boldsymbol{\nabla} p+\boldsymbol{\nabla} \cdot\left[2 \mu S-\frac{2 \mu(\boldsymbol{\nabla} \cdot \boldsymbol{U}) I}{3}\right]+f_{s t}+f_{g}
\end{gathered}
$$

Where $I$ is identity matrix, $p$ is pressure, $\mu$ is dynamic viscosity, $f_{s t}$ is surface tension force and $f_{g}$ is gravity force.

$$
S=0.5\left[\boldsymbol{\nabla} \cdot \boldsymbol{U}+(\boldsymbol{\nabla} \cdot \boldsymbol{U})^{T}\right]
$$

The continuity and momentum equations for incompressible flows are obtained by considering the changes in density of an infinitesimally small element as negligible or zero.

$$
\begin{gathered}
\boldsymbol{\nabla} \cdot(\boldsymbol{U})=0 \\
\rho\left(\frac{\partial \boldsymbol{U}}{\partial t}+\boldsymbol{U} \cdot \boldsymbol{\nabla} \boldsymbol{U}\right)=-\boldsymbol{\nabla} p+\boldsymbol{\nabla} \cdot\left[\mu\left(\boldsymbol{\nabla} \cdot \boldsymbol{U}+(\boldsymbol{\nabla} \cdot \boldsymbol{U})^{T}\right)\right]+\boldsymbol{f}_{s t}+\boldsymbol{f}_{g}
\end{gathered}
$$


In VOF, an interface capturing method, the location of interface is known based on the value of a scalar function called, liquid phase volume fraction. It is represented by $\alpha$

$$
\alpha=\frac{V_{l}}{V}
$$

Liquid phase volume fraction is 1 in liquid phase, 0 in gas phase and between these two values ( 0 and 1$)$ at interface. Its value is defined at the center of the cell. Mass of each phase is conserved when the transport equation of its phase fraction is satisfied. Liquid phase fraction is obtained by solving its transport equation given by

$$
\frac{\partial \alpha}{\partial t}+\boldsymbol{\nabla} \cdot\left(\boldsymbol{U}_{l} \alpha\right)=0
$$

Similarly, the transport equation of gas phase is

$$
\frac{\partial(1-\alpha)}{\partial t}+\boldsymbol{\nabla} \cdot\left(\boldsymbol{U}_{g}(1-\alpha)\right)=0
$$

It can be noted that the addition of liquid and gas phase fraction transport equations results in continuity equation.

Interface separating the phases is a numerical discontinuity in fluid properties. VOF method of modeling multiphase flows neglects the discontinuity and involves in obtaining a mixture representation of two or more phases. Velocity and transport properties of the mixture phase are obtained by volume averaging the velocities and properties of individual phases.

$$
\begin{gathered}
\boldsymbol{U}=\boldsymbol{U}_{l} \alpha+(1-\alpha) \boldsymbol{U}_{g} \\
\rho=\rho_{l} \alpha+(1-\alpha) \rho_{g} \\
\mu=\mu_{l} \alpha+(1-\alpha) \mu_{g}
\end{gathered}
$$

Continuity (Eq. 3.4) and momentum (Eq. 3.5) equations in VOF method are solved like those in single phase flows using the mixture phase properties given by Eq. 3.9 - Eq. 3.11. The surface tension force in the momentum equation is calculated based on Continuum 
Surface Force (CSF) formulation. Brackbill et al. developed this method to model surface tension in interfacial flows [46]. For a constant surface tension coefficient, surface tension force per unit volume is given by

$$
\boldsymbol{f}_{s t}=\sigma k\left(\frac{\nabla \alpha}{|\nabla \alpha|}\right)
$$

Where $\sigma$ is surface tension coefficient and $k$ is mean curvature of free surface

$$
k=-\nabla \cdot\left(\frac{\nabla \alpha}{|\nabla \alpha|}\right)
$$

\subsection{Phase fraction Equation in interFoam}

Liquid phase transport equation, Eq. 3.7 is an advection equation of scalar transport variable, liquid volume fraction. The discretization of this equation results in numerical diffusion irrespective of the scheme chose. So Eq. 3.7 is modified to reduce the numerical diffusion while introducing in OpenFOAM. Adding and subtracting the phase fraction flux in terms of mixture velocity, $\nabla \cdot(U \alpha)$, Eq 3.7 becomes

$$
\frac{\partial \alpha}{\partial t}+\boldsymbol{\nabla} \cdot\left(\boldsymbol{U}_{l} \alpha\right)+\boldsymbol{\nabla} \cdot(\boldsymbol{U} \alpha)-\boldsymbol{\nabla} \cdot\left(\left[\boldsymbol{U}_{l} \alpha+(1-\alpha) \boldsymbol{U}_{g}\right] \alpha\right)=0
$$

Here mixture phase velocity given by Eq. 3.9 is utilized. Rearranging the terms in Eq. 3.14 leads to

$$
\frac{\partial \alpha}{\partial t}+\boldsymbol{\nabla} \cdot(\boldsymbol{U} \alpha)+\boldsymbol{\nabla} \cdot\left(\left[\boldsymbol{U}_{l}(1-\alpha)-(1-\alpha) \boldsymbol{U}_{g}\right] \alpha\right)=0
$$

Interface artificial compression velocity, $U_{r}$ is the relative velocity between phases

$$
\boldsymbol{U}_{r}=\boldsymbol{U}_{l}-\boldsymbol{U}_{g}
$$

Eliminating $U_{g}, U_{l}$ in Eq. 3.15 and rearranging terms results in

$$
\frac{\partial \alpha}{\partial t}+\boldsymbol{\nabla} \cdot(\boldsymbol{U} \alpha)+\boldsymbol{\nabla} \cdot\left((1-\alpha) \boldsymbol{U}_{r} \alpha\right)=0
$$


In OpenFOAM, modified transport equation of phase volume fraction as indicated by Eq. 3.17 is solved. It can be observed that the artificial compression flux term, $\nabla$. $\left((1-\alpha) \boldsymbol{U}_{r} \alpha\right)$ provides additional surface compression and ensures the boundedness of phase fraction. This term vanishes in liquid and gaseous flow, but acts only at the interface. Thus, it limits numerical diffusion of interface without affecting the VOF solution. It's important to obtain an accurate value of liquid phase fraction as it determines the shape of the interface. The boundedness of liquid phase fraction between 0 and 1 is critical and the success of the code depends on ensuring the same.

\subsection{Evaporation Sub-Model}

Phase change in VOF is modeled using source terms in continuity, momentum and phase fraction equations along with the transport equation of temperature. In the current project, multiphase flows with three phases are considered. Liquid and its vapor phase along with surrounding air or gas are modeled. Vapor and gas are modeled as continuum phases with no interface separation between them. This continuum phase will be referred to as gaseous phase in this report. Vapor diffuses in gas, but both vapor and gas are insoluble in liquid phase. The bulk or advection based velocities of both gas and vapor phases are equal. Two

volume fraction variables are used to describe the presence of three phases. Liquid volume fraction is one only in liquid phase and vapor volume fraction is one only in vapor phase.

$$
\alpha_{1}= \begin{cases}0 & \text { In air or vapor phase } \\ 0<\alpha_{1}<1 & \text { At liquid interface } \\ 1 & \text { In liquid phase }\end{cases}
$$




$$
\alpha_{2}= \begin{cases}0 & \text { In air or liquid } \\ 0<\alpha_{2}<1 & \text { At liquid interface and zones of vapor diffused in air } \\ 1 & \text { In vapor phase }\end{cases}
$$

Transport properties like density $(\rho)$, thermal conductivity $(\lambda)$ of individual phases are volume averaged to obtain properties of single mixture phase.

$$
\begin{aligned}
\rho & =\alpha_{1} \rho_{l}+\alpha_{2} \rho_{v}+\left(1-\alpha_{1}-\alpha_{2}\right) \rho_{g} \\
\lambda & =\alpha_{1} \lambda_{l}+\alpha_{2} \lambda_{v}+\left(1-\alpha_{1}-\alpha_{2}\right) \lambda_{g}
\end{aligned}
$$

Specific heat at constant pressure is obtained by mass averaging the specific heats of individual phases.

$$
c_{p}=\rho_{l} \alpha_{1} c_{p, l}+\rho_{v} \alpha_{2} c_{p, v}+\rho_{g}\left(1-\alpha_{1}-\alpha_{2}\right) c_{p, g}
$$

Velocity is modeled as

$$
\boldsymbol{U}=\boldsymbol{U}_{l} \alpha_{1}+\boldsymbol{U}_{g p} \alpha_{2}+\boldsymbol{U}_{g p}\left(1-\alpha_{1}-\alpha_{2}\right)
$$

Or simply as

$$
\boldsymbol{U}=\boldsymbol{U}_{l} \alpha_{1}+\left(1-\alpha_{1}\right) \boldsymbol{U}_{g p}
$$

Where $\boldsymbol{U}_{g p}$ is velocity of gaseous phase.

Transport equations of liquid and vapor volume fractions have source terms to simulate reduction of mass from liquid and addition of mass to vapor phase during evaporation. If $\dot{m}^{\prime \prime \prime}$ represents the volumetric rate of mass transfer from liquid to vapor, the liquid phase fraction transport equation can be represented as Eq. 3.25 and the vapor phase fraction transport equation can be represented as Eq. 3.26

$$
\frac{\partial\left(\rho_{l} \alpha_{1}\right)}{\partial t}+\boldsymbol{\nabla} \cdot\left(\rho_{l} \boldsymbol{U}_{l} \alpha_{1}\right)=-\dot{m}^{\prime \prime \prime}
$$




$$
\frac{\partial\left(\rho_{v} \alpha_{2}\right)}{\partial t}+\nabla \cdot\left(\rho_{v} \boldsymbol{U}_{g p} \alpha_{2}\right)=\dot{m}^{\prime \prime \prime}+\boldsymbol{\nabla} \cdot\left(\rho_{g p} D_{v g} \boldsymbol{\nabla} \alpha_{2}\right)
$$

Where $D_{v g}$ is diffusivity of vapor in gas and $\rho_{g p}$ is density of vapor and gas phases together.

The negative rate of mass transfer in right hand side of Eq. 3.25 indicates that mass is removed from liquid phase during evaporation. Similarly, the positive rate of mass transfer in Eq. 3.26 indicates addition of mass in vapor phase. The gas phase fraction is calculated from liquid and vapor phase fractions and its transport equation is represented by

$$
\begin{gathered}
\frac{\partial\left(\rho_{g}\left(1-\alpha_{1}-\alpha_{2}\right)\right)}{\partial t}+\boldsymbol{\nabla} \cdot\left(\rho_{g} \boldsymbol{U}_{g p}\left(1-\alpha_{1}-\alpha_{2}\right)\right) \\
=\boldsymbol{\nabla} \cdot\left(\rho_{g} D_{v g} \boldsymbol{\nabla}\left(1-\alpha_{1}-\alpha_{2}\right)\right)
\end{gathered}
$$

Considering the flow as incompressible, the time rate of change of density is negligible or zero. The local time derivative and convective flux terms are expanded and arranged

$$
\begin{gathered}
\frac{\partial\left(\alpha_{1}\right)}{\partial t}+\boldsymbol{\nabla} \cdot\left(\boldsymbol{U}_{l} \alpha_{1}\right)=-\frac{\dot{m}^{\prime \prime \prime}}{\rho_{l}} \\
\frac{\partial\left(\alpha_{2}\right)}{\partial t}+\boldsymbol{\nabla} \cdot\left(\boldsymbol{U}_{g p} \alpha_{2}\right)=\frac{\dot{m}^{\prime \prime \prime}}{\rho_{v}}+\boldsymbol{\nabla} \cdot\left(D_{v g} \boldsymbol{\nabla} \alpha_{2}\right) \\
\frac{\partial\left(1-\alpha_{1}-\alpha_{2}\right)}{\partial t}+\boldsymbol{\nabla} \cdot\left(\boldsymbol{U}_{g p}\left(1-\alpha_{1}-\alpha_{2}\right)\right)=\boldsymbol{\nabla} \cdot\left(D_{v g} \boldsymbol{\nabla}\left(1-\alpha_{1}-\alpha_{2}\right)\right)
\end{gathered}
$$

The diffusion flux of gaseous phase (vapor and gas phase combined) is zero as it flows as a bulk fluid and it doesn't flow into liquid phase.

Addition of equations Eq. 3.28 to Eq. 3.30 and considering the volume averaged velocity equation Eq. 3.24 will result in continuity equation with source term

$$
\boldsymbol{\nabla} \cdot(\boldsymbol{U})=-\dot{m}^{\prime \prime \prime}\left(\frac{1}{\rho_{l}}-\frac{1}{\rho_{v}}\right)
$$


The volumetric rate of mass transfer from liquid phase to vapor phase can be calculated based on the diffusion rate of mass from high concentration to low concentration region. The liquid-vapor interface is a region of high concentration and vapor diffuses from liquid interface to the surroundings. The concentration gradient between the liquid and its vapor drives the mass diffusion from liquid to vapor phase. Based on Fick's law of mass diffusion, the diffusion flux of vapor is given by,

$$
\dot{m}_{v, d i f f}{ }^{\prime \prime}=-D_{v g} \rho_{g p} \frac{d Y_{v}}{d n}
$$

Where $Y_{v}$ is mass fraction of vapor and $n$ is the direction perpendicular to interface

Considering the bulk motion of gaseous phase, overall mass flux is given by

$$
\dot{m}_{v}^{\prime \prime}=Y_{v}\left(\dot{m}_{v}^{\prime \prime}+\dot{m}_{g}^{\prime \prime}\right)-D_{v g} \rho_{g p} \frac{d Y_{v}}{d n}
$$

Where $\dot{m}_{g}{ }^{\prime \prime}$ is total mass flux of gas phase.

Assuming the surrounding air or gas is insoluble in the liquid, its diffusion at the interface can be neglected.

$$
\dot{m}_{g}{ }^{\prime \prime}=0
$$

Adjusting the terms on Eq. 3.33 using Eq. 3.34

$$
\dot{m}_{v}^{\prime \prime}=-\frac{D_{v g} \rho_{g p}}{1-Y_{v}} \frac{d Y_{v}}{d n}
$$

Then the overall mass flux from liquid to vapor is given by

$$
\dot{m}^{\prime \prime}=-\dot{m}_{v}^{\prime \prime}
$$

This results in modification of Eq. 3.35

$$
\dot{m}^{\prime \prime}=\frac{D_{v g} \rho_{g p}}{1-Y_{v}} \frac{d Y_{v}}{d n}
$$


The mass flux obtained from Eq. 3.37 is only dependent on gradient of mass fraction. Mass fraction of vapor phase, $Y_{v}$, is calculating using vapor phase volume fraction, density of vapor and gaseous phases

$$
Y_{v}=\frac{\alpha_{2}}{1-\alpha_{1}} \frac{\rho_{v}}{\rho_{g p}}
$$

The interface between liquid and gaseous phases is assumed to be always at saturation state during phase change. Mass fraction of vapor at interface is given by

$$
Y_{v, s a t}=\frac{P_{v, s a t}}{P} * \frac{M_{v}}{M_{g p, s a t}}
$$

Where $P_{v \text {, sat }}$ is saturated vapor pressure, $P$ is static pressure, $M_{v}$ is molecular weight of vapor, $M_{g}$ is molecular weight of gas/air and $M_{g p \text {,sat }}$ is molecular weight of gaseous phase [16].

$$
M_{g p, s a t}=\frac{P_{v, s a t} * M_{v}+\left(P-P_{v, s a t}\right) * M_{g}}{P}
$$

Saturated vapor pressure is calculated using Wagner's equation given by

$$
\begin{aligned}
P_{v, \text { sat }}= & \frac{P_{c} * T_{c}}{T} \\
& *\left[a *\left(1-\frac{T}{T_{c}}\right)+b *\left(1-\frac{T}{T_{c}}\right)^{1.5}+c *\left(1-\frac{T}{T_{c}}\right)^{3}+d\right. \\
& \left.*\left(1-\frac{T}{T_{c}}\right)^{6}\right]
\end{aligned}
$$

Where $T_{c}$ is critical temperature and $P_{c}$ is critical pressure [16]

Energy transfer between two phases, which are in contact with each other at the interface, can be approximated to be due to thermal conduction between them. If the interface is assumed to be of thickness $d n$ in a direction normal to it, the heat flux across and normal to the interface is given by Fourier's law of conduction.

$$
\dot{q}^{\prime \prime}=-\lambda \frac{d T}{d n}
$$


The net heat flux to the liquid phase increases the temperature of liquid phase and the interface. The effect of radiation is not considered in this study. This increase in temperature of interface also increases the vapor pressure at the interface as obtained from Eq. 3.41. This process continues till the interface reaches particular temperature called saturation temperature, where the vapor pressure of the interface becomes equal to the vapor pressure of gaseous phase in its immediate surroundings. This results in a saturated condition of zero net mass transfer. The vapor mass fraction from Eq. 3.38 becomes 1 and the mass flux can't be calculated from Eq. 3.37. In such conditions, further heat transfer from surroundings to the interface results in slight increase of temperature at the interface and the vapor pressure at the interface becomes higher than that at the surroundings. This results in further mass transfer from liquid to the vapor phase. The amount of this additional mass transfer is obtained by considering energy and mass balance at the interface.

$$
\dot{m}^{\prime \prime}=-\frac{\lambda}{h_{v}} \frac{d T}{d n}
$$

Where $h_{v}$ is enthalpy of vaporization

This mass transfer from liquid to vapor phase results in reduction of temperature of liquid phase due to loss of enthalpy. This reduction in temperature to saturation conditions results in reduction of vapor pressure at the interface.

Based on Eq. 3.37 and Eq. 3.43

$$
\dot{m}^{\prime \prime}=\frac{D_{v g} \rho_{g p}}{1-Y_{v}} \frac{d Y_{v}}{d n}-\frac{\lambda}{h_{v}} \frac{d T}{d n}
$$

The volumetric rate of evaporation can be calculated from the mass flux using interface density, $\left|\nabla \alpha_{1}\right|[16]$.

$$
\dot{m}^{\prime \prime \prime}=\dot{m}^{\prime \prime}\left|\nabla \alpha_{1}\right|
$$

Applying Eq. 3.45 to Eq. 3.44 and extending the Eq. 3.44 to 3-dimensions using unit normal vector 


$$
\dot{m}^{\prime \prime \prime}=\frac{D_{v g} * \rho_{g p}}{1-Y_{v}} \nabla Y_{v} \cdot \frac{\nabla \alpha_{1}}{\left|\nabla \alpha_{1}\right|}\left|\nabla \alpha_{1}\right|-\frac{\lambda}{h_{v}} * \nabla T \cdot \frac{\nabla \alpha_{1}}{\left|\nabla \alpha_{1}\right|}\left|\nabla \alpha_{1}\right|
$$

The above equation of volumetric rate of mass transfer from liquid to vapor phase [47] simplifies to

$$
\dot{m}^{\prime \prime \prime}=\frac{D_{v g} * \rho_{g p}}{1-Y_{v}} \nabla Y_{v} \cdot \nabla \alpha_{1}-\frac{\lambda}{h_{v}} * \nabla T \cdot \nabla \alpha_{1}
$$

If interface is at saturation conditions, the mass fraction at the interface is 1 i.e. interface consists of pure vapor. This simplifies the equation of volumetric rate of mass transfer as

$$
\dot{m}^{\prime \prime \prime}=-\frac{\lambda}{h_{v}} \nabla T \cdot \nabla \alpha_{1}
$$

If the temperature at the interface is below saturation temperature, volumetric rate of mass transfer is simplified as

$$
\dot{m}^{\prime \prime \prime}=\frac{D_{v g} \rho_{g p}}{1-Y_{v}} \nabla Y_{v} \cdot \nabla \alpha_{1}
$$

Energy equation is introduced to model the effect of heat transfer. The source term in the energy equation is the heat transferred due to mass transfer during evaporation.

$$
\frac{\partial\left(\rho c_{p} T\right)}{\partial t}+\nabla \cdot\left(\rho \boldsymbol{U} c_{p} T\right)=\boldsymbol{\nabla} \cdot(\lambda \boldsymbol{\nabla} T)+h_{v} \dot{m}^{\prime \prime \prime}
$$

The temperature at the interface is constrained to saturation temperature and surface superheat is not considered.

\subsection{Phase fraction Equations in evapFoam}

The artificial interface compression flux term is introduced and individual phase velocities are eliminated from Eq. 3.28 and Eq. 3.29 in a similar fashion as done in Eq. 3.17 of interFoam. The modified set of equations are given by

$$
\frac{\partial \alpha_{1}}{\partial t}+\boldsymbol{\nabla} \cdot\left(\boldsymbol{U} \alpha_{1}\right)+\boldsymbol{\nabla} \cdot\left(\left(1-\alpha_{1}\right) \boldsymbol{U}_{r} \alpha_{1}\right)=-\frac{\dot{m}^{\prime \prime \prime}}{\rho_{l}}
$$




$$
\frac{\partial \alpha_{2}}{\partial t}+\boldsymbol{\nabla} \cdot\left(\boldsymbol{U} \alpha_{2}\right)-\boldsymbol{\nabla} \cdot\left(\alpha_{2} \boldsymbol{U}_{r} \alpha_{1}\right)=\frac{\dot{m}^{\prime \prime \prime}}{\rho_{v}}+\boldsymbol{\nabla} \cdot\left(D_{v g} \boldsymbol{\nabla} \alpha_{2}\right)
$$

The source terms in the phase transport equations are modified using the continuity equation to ensure the numerical boundedness of the source and sink terms.

Adding and subtracting $\alpha_{1}(\nabla \cdot U)$ in the RHS of the Eq. 3.51

$$
\begin{aligned}
\frac{\partial \alpha_{1}}{\partial t}+\boldsymbol{\nabla} \cdot\left(\boldsymbol{U} \alpha_{1}\right) & +\boldsymbol{\nabla} \cdot\left(\left(1-\alpha_{1}\right) \boldsymbol{U}_{r} \alpha_{1}\right) \\
= & -\frac{\dot{m}^{\prime \prime \prime}}{\rho_{l}}+\alpha_{1}(\boldsymbol{\nabla} \cdot \boldsymbol{U})-\alpha_{1}(\boldsymbol{\nabla} \cdot \boldsymbol{U})
\end{aligned}
$$

Using the definition of continuity equation Eq. 3.31

$$
\begin{aligned}
& \frac{\partial \alpha_{1}}{\partial t}+\boldsymbol{\nabla} \cdot\left(\boldsymbol{U} \alpha_{1}\right)+\boldsymbol{\nabla} \cdot\left(\left(1-\alpha_{1}\right) \boldsymbol{U}_{r} \alpha_{1}\right) \\
& =-\frac{\dot{m}^{\prime \prime \prime}}{\rho_{l}}+\alpha_{1}(\boldsymbol{\nabla} \cdot \boldsymbol{U})+\alpha_{1} \dot{m}^{\prime \prime \prime}\left(\frac{1}{\rho_{l}}-\frac{1}{\rho_{v}}\right) \\
& \frac{\partial \alpha_{1}}{\partial t}+\boldsymbol{\nabla} \cdot\left(\boldsymbol{U} \alpha_{1}\right)+\boldsymbol{\nabla} \cdot\left(\left(1-\alpha_{1}\right) \boldsymbol{U}_{r} \alpha_{1}\right) \\
& =\alpha_{1}(\boldsymbol{\nabla} \cdot \boldsymbol{U})-\dot{m}^{\prime \prime \prime}\left(\frac{1}{\rho_{l}}-\alpha_{1}\left(\frac{1}{\rho_{l}}-\frac{1}{\rho_{v}}\right)\right)
\end{aligned}
$$

Similarly

$$
\begin{aligned}
\frac{\partial \alpha_{2}}{\partial t}+\boldsymbol{\nabla} \cdot( & \left(\boldsymbol{U} \alpha_{2}\right)-\boldsymbol{\nabla} \cdot\left(\alpha_{2} \boldsymbol{U}_{r} \alpha_{1}\right) \\
& =\boldsymbol{\nabla} \cdot\left(D_{v g} \boldsymbol{\nabla} \alpha_{2}\right)+\alpha_{1}(\boldsymbol{\nabla} \cdot \boldsymbol{U})+\dot{m}^{\prime \prime \prime}\left(\frac{1}{\rho_{v}}+\alpha_{1}\left(\frac{1}{\rho_{l}}-\frac{1}{\rho_{v}}\right)\right)
\end{aligned}
$$

Momentum equation is not affected by the evaporation sub-model and Eq. 3.5 is used in evapFoam too. No source terms are added to momentum equation as their effect is already introduced in continuity equation 


\subsection{Interface Reconstruction}

Geometric interface reconstruction schemes like Piecewise Linear Interface Calculation (PLIC) involve explicit interface reconstruction and provide a sharp interface. But they can only be implemented on a structured hexahedral grid. Algebraic VOF implemented in OpenFOAM is simper and can be used on complex geometries and unstructured grids. It doesn't involve any reconstruction of interface and hence results in poor shape preservation. It involves solving of discretized form of phase fraction partial differential equations using algebraic differencing schemes to result in face centered phase fractions. The choice of bounded compressive advection schemes for spatial discretization is critical as lower order schemes result in smearing and higher order schemes may result in wrinkling or numerical oscillations of the interface.

\subsection{MULES}

In all the multiphase solvers of OpenFOAM, an explicit advection scheme called Multidimensional Limiter with Explicit Solution (MULES) is used to ensure the boundedness of phase fraction terms. Pressure - velocity equation is coupled with the transport equation of phase fraction. Phase fraction fluxes are calculated based on the velocity obtained in the predictor step and then in the correction step phase fraction values are limited from falling below 0 and shooting above 1. MULES is generally an explicit scheme and it requires courant number limit to be adhered to. A new semi implicit variant of MULES which limits explicit MULES to only corrector step in conjunction with the implicit predictor step can also be used. It is faster than the traditional explicit MULES.

\subsection{Development of evapFoam}

The mathematical model as described in sections 3.3 and 3.4 is written in $\mathrm{C}++$ and implemented as a new solver, evapFoam, in OpenFOAM-2.3.x package. It is compiled with gcc-4.8.2, a $\mathrm{C}++$ compiler. It is developed based on a built-in solver, interPhaseChangeFoam and uses a combination of existing and modified libraries. The 
existing solver models two phase flows of incompressible, isothermal and immiscible fluids with phase change based on cavitation phenomenon.

\section{evapFoam.C}

This file contains the main code of the solver and is the only compilable file in the solver. It is the first file that runs when solver is invoked. This file doesn't explicitly contain any equations discussed earlier, but it can call the .H files in which these equations are written. This file starts by reading time, mesh and calculating time-step based on CFL condition. In every time-step, it updates the mixture properties and interface based on the information from the previous time step. It then calls the necessary files required for calculation of liquid and vapor phase fractions, velocity temperature and pressure. In a Pressure-Velocity predictor corrector loop, velocity initially solved will be used to calculate pressure and then the velocity initially used is corrected.

\section{alphaEqnSubCycle.H}

This file first calculates the interface compression flux term used in Eq. 3.55 and then calls alphaEqn.H where liquid and vapor phase fraction equations Eq. 3.55 and Eq. 3.56 are solved.

\section{$\underline{\text { alphaEqn.H }}$}

Liquid volume fraction file 'alphaEqn.H' is modified to accommodate transport equations of liquid and vapor volume fractions Eq. 3.55 and Eq. 3.56. The temporary explicit flux of liquid and vapor phase fractions tPhiAlpha1 and tPhiAlpha2 are first obtained by neglecting the compression flux and then corrected fluxes are calculated by considering them. It should be noted that the compression flux is used only for liquid phase fraction. The corrected fluxes due to liquid and vapor phase fractions are used in MULES to obtain bounded liquid and vapor phase fraction values. The source terms in Eq. 3.55 and Eq. 3.56 are obtained from phase change model. All mixture transport properties are re-calculated 
based on the updated values of liquid and vapor phase fractions. It can be noted that the continuity equation Eq. 3.31 is not solved explicitly. The continuity equation is substituted in deriving Eq. 3.55 and hence solving for phase fractions implicitly satisfies the continuity equation.

\section{$\underline{\text { UEqn.H}}$}

This file contains the momentum equation. Unlike alphaEqn.H, this file doesn't solve for velocity. Pressure - Velocity coupling requires that velocity is solved along with pressure in pEqn.H.

\section{pEqn.H}

In this file, velocity is predicted first and the flux phiHbyA is created. Then the flux phig is calculated by considering the forces from surface tension and gravity along with the flux from velocity. Flux due to phase change is also considered. This is required because the initial predicted velocity did not consider the sources as given by the continuity equation Eq. 3.31. All the flux terms are used to calculate the pressure term which in turn is used to obtain the corrected velocity in terms of corrected velocity flux.

\section{$\underline{\text { TEqn.H}}$}

The energy equation Eq. 3.50 is introduced in 'TEqn.H' file and is solved for temperature. Thermo-physical properties are updated before solving for temperature field using volume fraction values calculated in the current time-step.

\section{Phase Change Model}

A phase change model is built for three phase mixture flows where volumetric mass flow rate equation Eq. 3.43 is solved. It contains additional equations, Eq. 3.44 to Eq. 3.47 needed to calculate volumetric mass flow rate. This model replaces the cavitation models in interPhaseChangeFoam. OpenFOAM provides a base class phaseChangeTwoPhaseMixture for calculation of source terms. It is modified to introduce three phases. Source terms in phase fraction equations are created and thermophysical 
properties are read. Mass flow terms are modeled as a pair, but only evaporation is defined. Condensation terms are set as zero.

\section{$\underline{\text { Libraries }}$}

Source codes of immiscible, incompressible flows. two phase mixtures and interface properties are modified to model thermophysical properties as given by Eq. 3.20 - Eq. 3.22 based on volume fractions of three phases.

\section{Application of evapFoam}

To run a simulation using evapFoam, the case should contain all the input required for the solver. This includes thermophysical properties of all three phases along with the properties need for calculation of volumetric mass flow rate. It also needs the initial values of phase fractions of liquid, vapor along with the domain temperature. Any case in OpenFOAM can be run in parallel irrespective of the solver used. The computational domain is split into a pre-defined number of cases using decomposePar. At the end of the simulation, the split domains must be re-assembled using reconstructPar to obtain the solution of the whole domain. The computational time is significantly reduced using parallel processing.

\subsection{Limitations}

Though it was intended to develop a model that could give results of experimental quality, certain limitations on the solver and in the general capabilities of OpenFOAM prevent it from doing so.

- Current solver doesn't consider superheat at the interface. The temperature of surface is limited to saturated temperature and doesn't exceed this value during the phase change.

- It is applicable only for incompressible flows. VOF method implemented in OpenFOAM simulates only incompressible immiscible flows. 
- Thermo-physical properties are considered to be constant throughout the simulation. Properties like density and specific heat are temperature dependent. But the current solver doesn't take the effect of temperature on the properties.

- It is currently applicable for single component liquids only. It doesn't have a provision to consider multi-component liquid based on their percentage of composition. 


\section{CHAPTER 4 RESULTS AND DISCUSSION ${ }^{1}$}

The results of simulations of this project are presented in three parts. In the first part, the built-in VOF model in OpenFOAM is validated with simulations of binary droplet impingement and droplet impingement on wet wall. Both the simulations are performed under isothermal conditions. In the second part, the evaporation model implemented in VOF is validated with 3-dimensional simulations of droplets in almost static hot environment and droplet in cross-stream of air. In the third part, the evaporation solver is applied to simulate multiple-droplet impingement on a hot wall.

\subsection{VOF Without Evaporation}

\subsubsection{Binary Droplet Impingement}

The collision of two droplets of equal size D moving towards each other at a relative velocity $2 \mathrm{U}$ is studied. The outcome of the binary droplet collision is primarily influenced by process parameters given by non-dimensional numbers such as Weber number, Reynolds number and Impact parameter. The physical properties of both the phases are listed in the table below.

\footnotetext{
${ }^{1}$ The material contained in this chapter was previously published as a technical paper in Society of Automotive Engineers

Potham, S., Zhao, L., and Lee, S., "Numerical Study on Evaporation of Spherical Droplets Impinging on the Wall Using Volume of Fluid (VOF) Model," SAE Technical Paper 2017-01-0852, 2017, doi:10.4271/2017-01-0852. Reprinted with permission Copyright 2017 C SAE International Further distribution of this material is not permitted without prior permission from SAE.
} 
Table 1: Thermo-physical properties of phases

\begin{tabular}{|c|c|c|c|}
\hline Phase & $\begin{array}{l}\text { Surface tension } \\
(\mathrm{N} / \mathrm{m})\end{array}$ & Density $\left(\mathrm{kg} / \mathrm{m}^{3}\right)$ & $\begin{array}{c}\text { Kinematic } \\
\text { viscosity }\left(\mathrm{m}^{2} / \mathrm{s}\right)\end{array}$ \\
\hline Air & - & 1.2 & $1.48 * 10^{-5}$ \\
\hline Water & 0.072 & 1000 & $0.1 * 10^{-5}$ \\
\hline
\end{tabular}

The droplets are confined in a three-dimensional domain of 6.25 D X 3.75 D X 3.75 D and are initially separated by a distance $\mathrm{D}$. The domain is filled with air at standard atmospheric pressure and temperature. Two cases of droplet collision are presented in this study. Case 1 presents head-on collision and Case 2 presents off-axis collision. In head-on collision, the velocity vectors of both the droplets are at $180^{\circ}$ angle and are parallel to the line joining the centers of the droplets. In off-axis simulation, the line joining the centers of the droplets is at an angle with the relative velocity vector placed at the center of the other droplet. The simulation case set up details for both head-on and off-axis collision are presented below

Table 2: Droplet Collisions - Case setup details

\begin{tabular}{|c|c|c|}
\hline & Case 1 & Case 2 \\
\hline Type of collision & Head on & Off axis \\
\hline Domain dimensions & $5 \mathrm{~mm} \mathrm{X} \mathrm{3} \mathrm{mm} \mathrm{X} \mathrm{3} \mathrm{mm}$ & $5 \mathrm{~mm} \mathrm{X} \mathrm{3} \mathrm{mm} \mathrm{X} \mathrm{3} \mathrm{mm}$ \\
\hline Droplet diameter & $0.8 \mathrm{~mm}$ & $0.8 \mathrm{~mm}$ \\
\hline Grid size & $16.66 \mu \mathrm{m}$ & $28.5 \mu \mathrm{m}$ \\
\hline Relative velocity & $1.9 \mathrm{~m} / \mathrm{s}$ & $2.72 \mathrm{~m} / \mathrm{s}$ \\
\hline Ambient pressure & $1 \mathrm{~atm}$ & Water (alpha=1) \\
\hline Phase 1 & Water (alpha=1) & Air (alpha $=0$ ) \\
\hline Phase 2 & Air (alpha $=0)$ & 0.43 \\
\hline Weber number & 40 & Stretching separation \\
\hline Impact parameter & 0 & \\
\hline Results & Separation with a satellite \\
& droplet formation & \\
\hline
\end{tabular}




\section{Case 1:}

The domain is meshed with uniform grid size of $\mathrm{D} / 50$. Water droplets are considered for this case. Experimental results of Ashgriz and Poo are used for comparison with simulation results. One case of head-on collision with equal sized droplets is considered for the simulation. Both droplets are modeled with equal initial diameter of $800 \mu \mathrm{m}$ and velocity of $0.95 \mathrm{~m} / \mathrm{s}$ towards each other. Weber number calculated based on the relative velocity between droplets is 40 and Reynolds number is 1520. It was observed that the collision led to coalescence, followed by reflexive separation with a satellite droplet formation. The time sequence of shape evolution of the liquid phase during the process of collision is shown in Fig. 1. It can be concluded that the simulation results present a great deal of similarity with the experimental results.

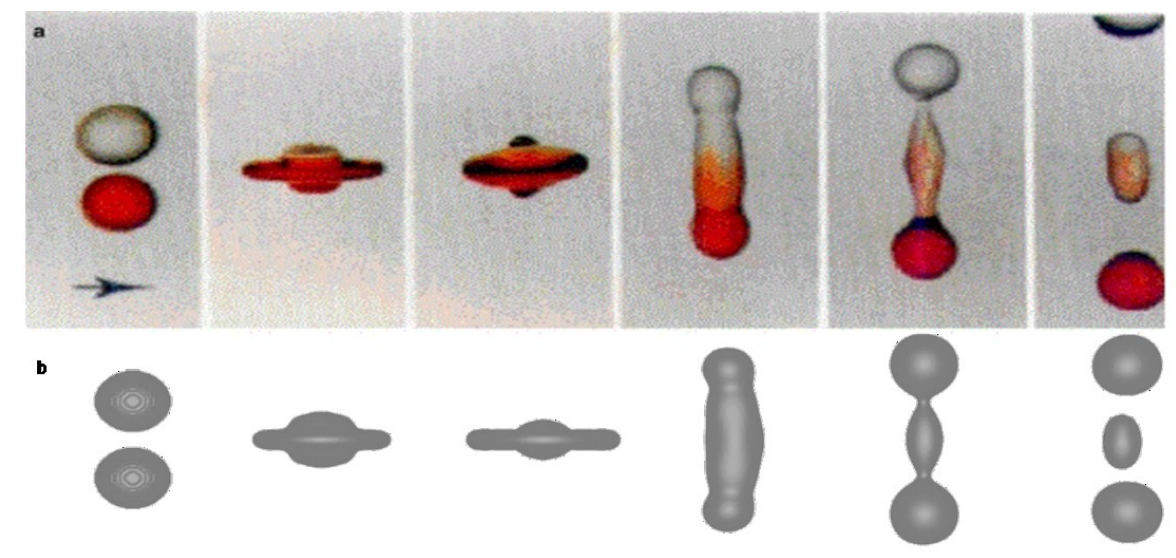

Figure 1: Head-on collision of Water droplets at We-40 from a) Ashgriz and Poo (1990) ${ }^{2}$ b) Simulation.

\section{Case 2:}

In this case, the domain is meshed with a mesh size of $\mathrm{D} / 28$ and dynamic mesh refinement of level 2 is used. The water droplets collide with an impact parameter 0.43 and weber number 83 . The initial velocities of both the droplets is assumed to be at $180^{\circ}$ to each other.

\footnotetext{
${ }^{2}$ Reprinted by permission of Cambridge University Press, N. Ashgriz and Y.J. Poo, Coalescence and Separation in binary collisions of liquid drops, Journal of Fluid Mechanics 221 (1990) 183-204
} 
The collision results in coalescence of the droplets initially, followed by stretching separation and satellite droplets formation. The experimental results show a thin membrane during the stretching phase, whereas simulation results indicate that the membrane is broken with a small droplet formation.

The satellite droplets formed in the simulation are of unequal size whereas the experiment shows droplets of almost equal size. The thin membrane couldn't be accurately simulated. The discrepancy may be due to differences in pinch off point in experiment and simulation. The position of pinch off point and the shape of the elongated coalesced droplet is influenced by the direction of initial velocities. Inspite of some differences, the outcome of off-axis collision is reasonably well predicted by the simulation.

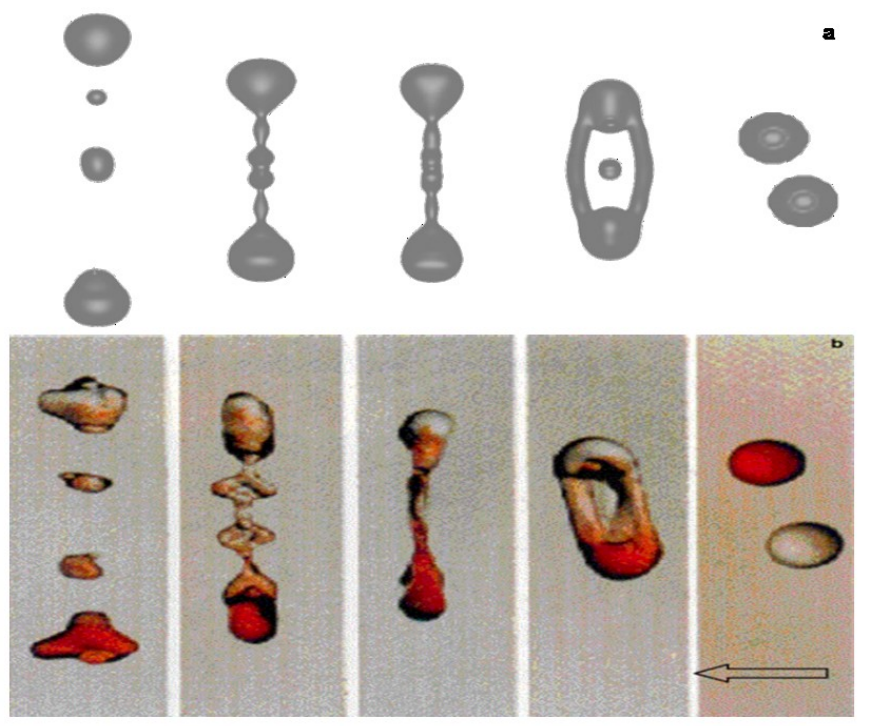

Figure 2: Time evolution of droplet impingement at impact parameter 0.4 a) Simulation b)Ashgriz and $\operatorname{Poo}(1990)^{3}$

\footnotetext{
${ }^{3}$ Reprinted by permission of Cambridge University Press, N. Ashgriz and Y.J. Poo, Coalescence and Separation in binary collisions of liquid drops, Journal of Fluid Mechanics 221 (1990) 183-204
} 


\subsubsection{Droplet Impingement On Wet Wall}

A water droplet impinging on a thin film of water on a wall is simulated in a threedimensional domain filled with air at standard atmospheric pressure and temperature. The domain is of 4D X 4D X 4D with the fine grid size of $\mathrm{D} / 80$.

The temperature and pressure are uniform throughout the domain initially. The case is isothermal and hence no loss of liquid phase is expected. Droplet diameter of $9 \mathrm{~mm}$, with Weber number 598, Reynolds number 17467 and film thickness of $1 \mathrm{~mm}$ are used. As the droplet approached the wall with a uniform velocity, it interacts with the surrounding air. This leads to a slight elongation of the droplet in vertical direction resulting in oval shape. At the impact, a capillary wave is created on the liquid film and it travels to the boundaries of the domain.

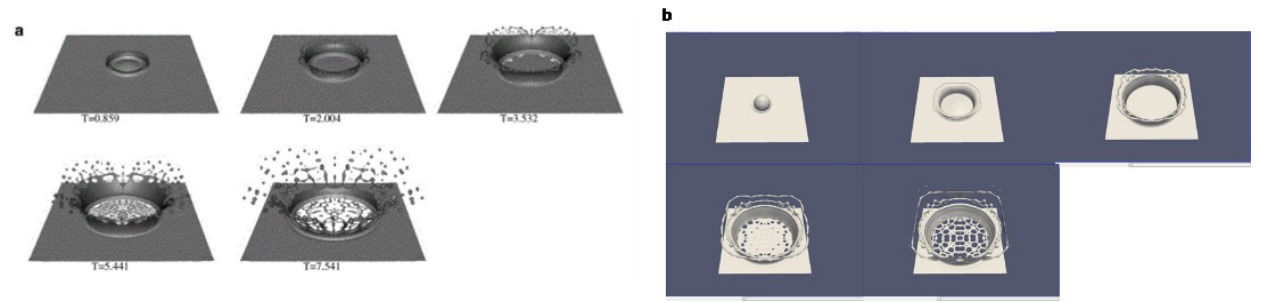

Figure 3: Temporal evolution of water droplet impinging on a wet wall a)Published ${ }^{4}$ b) Simulation Initially, after the impact of the droplet, liquid on the film splashes with a crown formation. The height of the crown gradually increases over time and the top portion of the crown detaches to form a ring. Due to the momentum of the splash, the detached ring continues to expand its diameter and finally break into numerous small droplets. In the meantime, another ring detaches from the crown and the process would repeat till the splash subsides. The simulation catches the process of splash evolution correctly except for the breakup of ring into numerous small droplets. The simulation indicates only a few droplets contrary to the result of Nikolopoulos et al. [36].

\footnotetext{
${ }^{4}$ Reprinted by permission of Elsevier, N. Nikolopoulos, A. Theodorakakos, G. Bergeles, Threedimensional numerical investigation of a droplet normally onto a wall film, Journal of Computational Physics 225 (1) (2007) 322-341
} 


\subsection{VOF With Evaporation}

\subsubsection{Single droplet evaporation}

In VOF method, the flow equations are discretized on the three-dimensional grid in space. It's accuracy is highly dependent on the mesh resolution. Physically, interface separating the phases is infinitesimally thin. To model such thin interface computationally, a very fine grid is required. In VOF methods, increasing the mesh resolution could only improve the accuracy of the simulation. However, the usage of computationally expensive mesh resolution is limited by the availability of computational resources and time. A fine balance needs to be stuck between the level of accuracy desired and the computational expense needed to achieve that. This section presents a mesh dependency study that has been conducted to understand the effect of grid size on the results of simulations of evaporation of single droplet. Three different grid sizes of $\mathrm{D}_{0} / 15, \mathrm{D}_{0} / 18, \mathrm{D}_{0} / 20$ are studied, where $\mathrm{D}_{0}$ is initial droplet diameter. In each case, the initial and boundary conditions are maintained the same. The initial diameter of water droplet is chosen $100 \mu \mathrm{m}$ in a quiescent threedimensional domain of $400 \mu \mathrm{m} \times 400 \mu \mathrm{m} \times 400 \mu \mathrm{m}$. The domain is filled with hot air at a temperature of $646 \mathrm{~K}$ with microgravity conditions and droplet at saturation temperature of $373 \mathrm{~K}$ is present at the center of the domain [47]. Heat from the droplet surroundings at higher temperature is transferred to the droplet at lower temperature supplying sensible and latent heat required for droplet heating and evaporation. The mass transfer from the liquid droplet to vapor phase results in reduction of droplet diameter over time. The differential equation for change in droplet diameter in this process is given by the $\mathrm{D}^{2}$ law is

$$
\frac{d\left(D^{2}\right)}{d t}=-\frac{8 \lambda_{g}}{\rho_{l} c_{p, g}}\left(\frac{c_{p, g}\left(T_{\infty}-T_{s a t}\right)}{\Delta h_{v}}+1\right)
$$

The analytical solution of Eq. 4.1 is given by Eq. 4.2 by taking the assumption that the process is at steady state at any given instant of time, $t$.

$$
D^{2}(t)=D_{0}^{2}-K t
$$


Where,

$$
K=\frac{8 \lambda_{g}}{\rho_{l} c_{p, g}}\left(\frac{c_{p, g}\left(T_{\infty}-T_{s a t}\right)}{\Delta h_{v}}+1\right)
$$

The ratio of squares of droplet diameter at given time to droplet diameter at start of simulation is plotted in Fig. 4 against time in all three simulation cases and compared with the solution obtained from $\mathrm{D}^{2}$ law. It was observed that the accuracy of the simulation compared to the published analytical results is dependent on the mesh resolution. The degree of the mesh resolution is limited by the computational power and time. A grid resolution of $\mathrm{D} / 20$ was observed to give better results and was adopted for the remaining portion of this study. It can be observed that the result obtained with fine mesh grid is of reasonable accuracy inspite of the assumption of the mathematical model used to develop the solver. This is due to a similar set of assumptions involved in obtaining the $\mathrm{D}^{2}$ law. Analytical solution is obtained by considering thermophysical properties at initial temperature of the droplet and are constant with changes in temperature. It also assumes that the interface is saturated and mass fraction at the interface is obtained by considering saturated vapor pressure corresponding to the droplet surface temperature.

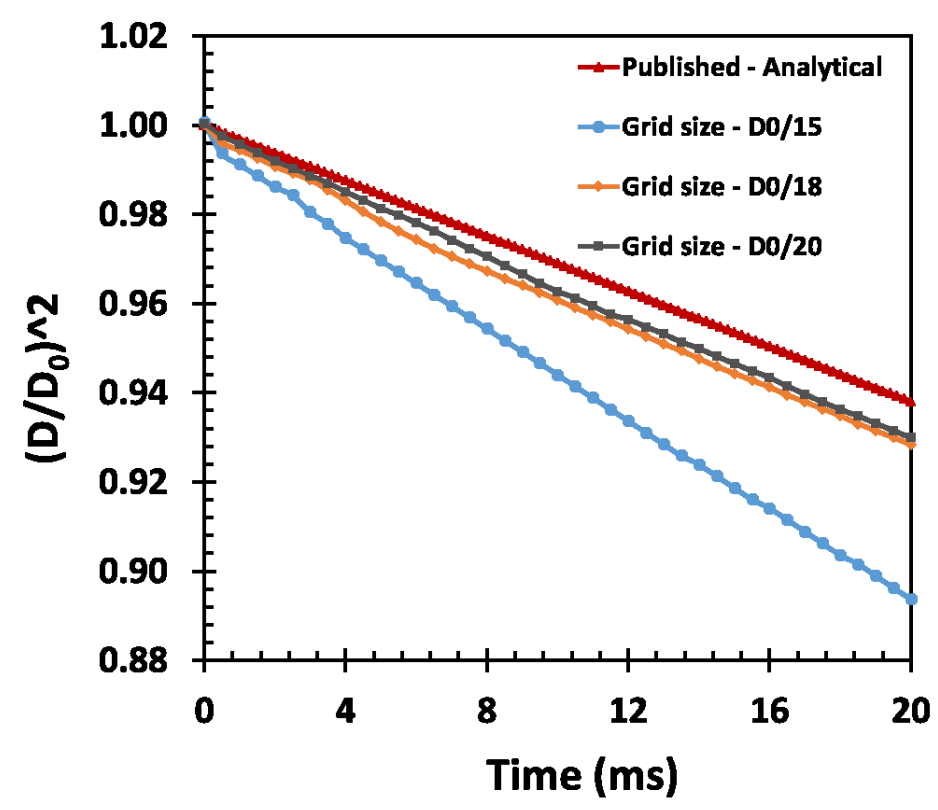

Figure 4: Effect of grid resolution on simulation results. 


\subsubsection{Droplet in cross-stream of hot air}

A water droplet of $2.1 \mathrm{~mm}$ diameter at a temperature of $343 \mathrm{~K}$ in cross-stream of hot air at temperature of $363 \mathrm{~K}$ and velocity of $15 \mathrm{~m} / \mathrm{s}$ is simulated in a three-dimensional domain of size $10 \mathrm{~mm}$ x $4 \mathrm{~mm} \times 4 \mathrm{~mm}$. The left side face of the domain is modelled as an inlet and right side face as outlet. Air velocity is uniform across the cross-section at the inlet. The pressure conditions at the outlet are unknown and so the velocity at the outlet is modeled such that it can be either towards outlet or towards inlet. The lateral faces are modeled as walls with free slip condition. The phase change phenomenon with the transfer of mass of the droplet from liquid phase to vapor phase and the transfer of heat from surrounding air to liquid and vapor phases are mainly influenced by the thermophysical properties of the droplet, temperature and vapor concentration fields around the droplet. The temperature and vapor concentration fields in the domain obtained by using the newly developed evapFoam solver are presented in Fig. 6 and Fig. 5 respectively and are in qualitative agreement with the simulation results obtained for a similar case by Schlottke and Weigand [16].

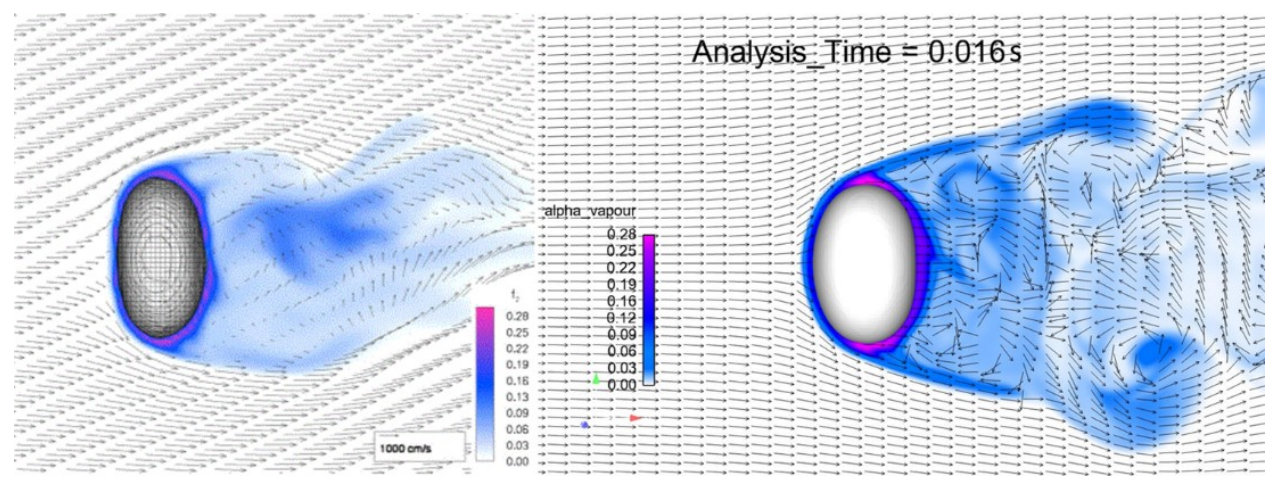

Figure 5: Vapor fraction distribution around the droplet at 16 ms. Published ${ }^{5}$ (left), Simulation (right).

The droplet deforms from its spherical shape in the beginning to an oblate shape. The deformed droplet relaxes back to its spherical shape due to cohesive forces on the surface

\footnotetext{
${ }^{5}$ Reprinted by permission of Elsevier, J. Schlottke, B. Weigand, Direct Numerical Simulation of evaporating droplets, Journal of Computational Physics 227 (10) (2008) 5215-5237
} 
of the droplet. The solver was able to simulate the oscillations of droplet diameter with decreasing amplitude. It was observed that the flow behind the droplet consists of vortices with low velocity and few pockets of high vapor concentration. The vapor fraction distribution shows a high level of vapor concentration on the surface of the droplet, followed by the vicinities of the vortices behind the droplet. The high vapor concentration zones behind the droplet is due to inadequate mixing with the surrounding air. The less density of the arrows, indicting lower velocity of the surrounding air, in the wake of the droplets causes less mixing with the vapor.

The temperature distribution shown in Fig. 6 indicates that the droplet is at a lower temperature compared to the surrounding air and the temperature of the droplet shows gradual gradient normal to the surface. It can also be observed that the high vapor concentration zones are at lower temperature compared to the zones that didn't mix with vapor.
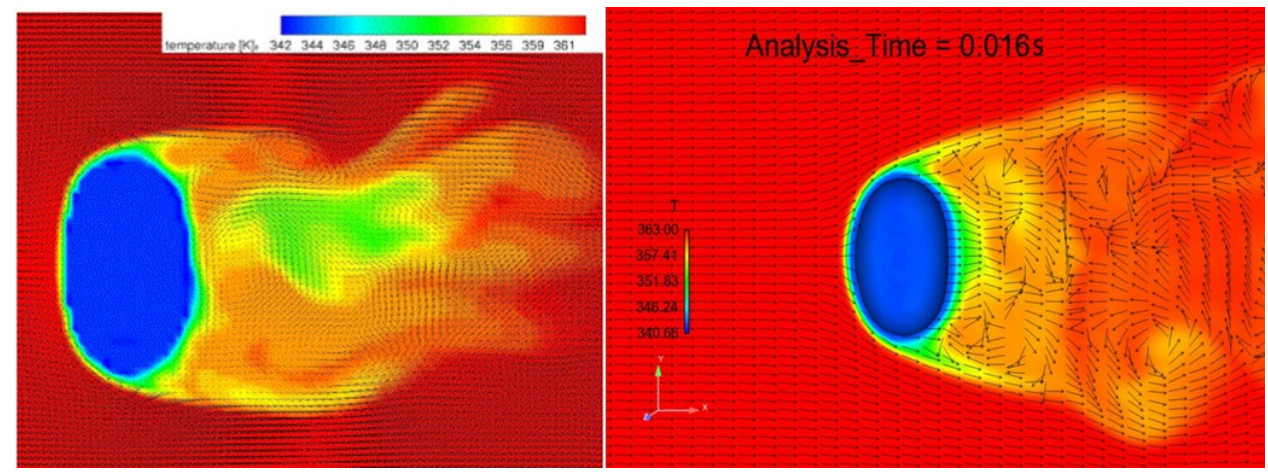

Figure 6: Temperature distribution around the droplet -Published result (left) ${ }^{6}$, Current simulation (right)

\footnotetext{
${ }^{6}$ Reprinted by permission of Elsevier, J. Schlottke, B. Weigand, Direct Numerical Simulation of evaporating droplets, Journal of Computational Physics 227 (10) (2008) 5215-5237
} 


\subsection{Multiple Droplet Impingement On Hot Wall}

The evapFoam solver developed in this project is applied to study the effect of droplet number and arrangement on evaporation during multiple droplet impingement on hot wall. Three cases are chosen for this study and all of them are in film boiling regime. The temperature of wall in each case is above the Leidenfrost temperature of n-heptane in this conditions

\section{Case setup}

The description and boundary conditions of each case are discussed in this section. The arrangement of $n$-heptane droplets in each case is presented in Fig. 7. The blue colored circles indicate droplets and the grey rectangle indicates the wall. The redo colored dotted line indicates the cut-plane, whose front view is used Fig. 9. It should be noted that all droplets in any case are all of equal diameter and are separated from each other by a distance equal to radius of the droplet in that particular case. The sum of masses of all droplets in any case is constant and the diameter of the droplets varies from case to case. The initial vertical distance between any droplet center and the wall is the same. The droplets fall with an initial velocity of $0.8 \mathrm{~m} / \mathrm{s}$. The initial temperature and pressure of the domain including the droplet is $298 \mathrm{~K}$ and $1 \mathrm{~atm}$. The $\mathrm{n}$-heptane droplets with Leidenfrost temperature of $473 \mathrm{~K}$ impinge on wall maintained at a constant temperature of $483 \mathrm{~K}$. Hence, the evaporation of the droplets is in film boiling regime. The contact angle between the droplet and the hot wall is taken as $120^{\circ}$ [40]. 


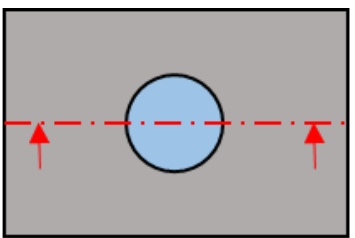

Single droplet (Top view)

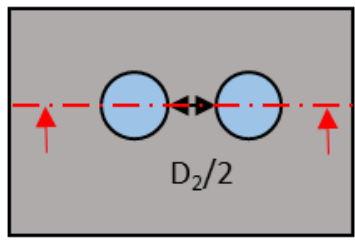

2 droplets (Top view)

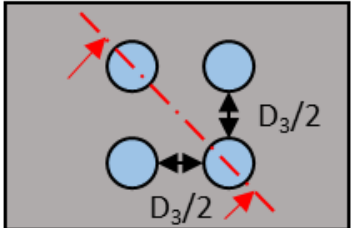

4 droplets (Top view)
Case 1

\section{Single droplet (Front view)}

Case 2

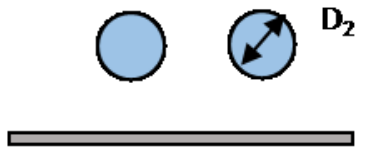

2 droplets (Front view)

Case 3

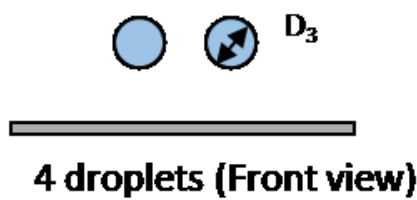

Figure 7: Droplet arrangement of three cases

The geometric details and process parameters of cases examined are presented in Table 1

Table 3: Simulation parameters of three cases.

\begin{tabular}{|c|c|c|c|}
\hline & Case 1 & Case 2 & Case 3 \\
\hline Liquid & n-heptane & n-heptane & n-heptane \\
\hline No. of droplets & 1 & 2 & 4 \\
\hline Diameter & $1.5 \mathrm{~mm}$ & $1.19 \mathrm{~mm}$ & $0.944 \mathrm{~mm}$ \\
\hline Velocity & $0.8 \mathrm{~m} / \mathrm{s}$ & $0.8 \mathrm{~m} / \mathrm{s}$ & $0.8 \mathrm{~m} / \mathrm{s}$ \\
\hline Weber number & 41 & 32.6 & 25.9 \\
\hline Reynolds number & 3750 & 2975 & 2360 \\
\hline
\end{tabular}

A n-heptane droplet is at the center of the domain surrounded by dry air at the beginning of the simulation. The domain used in this study is three-dimensional of size $8 \mathrm{~mm} \times 5 \mathrm{~mm}$ $\mathrm{x} 5 \mathrm{~mm}$ and is filled with air at atmospheric pressure and temperature of $298 \mathrm{~K}$. The domain is meshed with a non-uniform sized grid with a minimum size of $50 \mu \mathrm{m}$ in $\mathrm{x}, \mathrm{z}$ directions and $7.5 \mu \mathrm{m}$ in $\mathrm{y}$ direction. The maximum grid size in $\mathrm{x}$ and $\mathrm{z}$ directions is $200 \mu \mathrm{m}$ and 613 
$\mu \mathrm{m}$ in $\mathrm{y}$ direction. A fine mesh is used in the center of the domain and near the hot wall where droplets contact and spread on the wall. The heat conduction to the droplet is maximum in this zone and droplet shape after impact is also dependent upon grid resolution in this region. The mesh used for the Case 1 in the current study is presented in Fig. 8. A similar grid is used for Case 2 and Case 3.

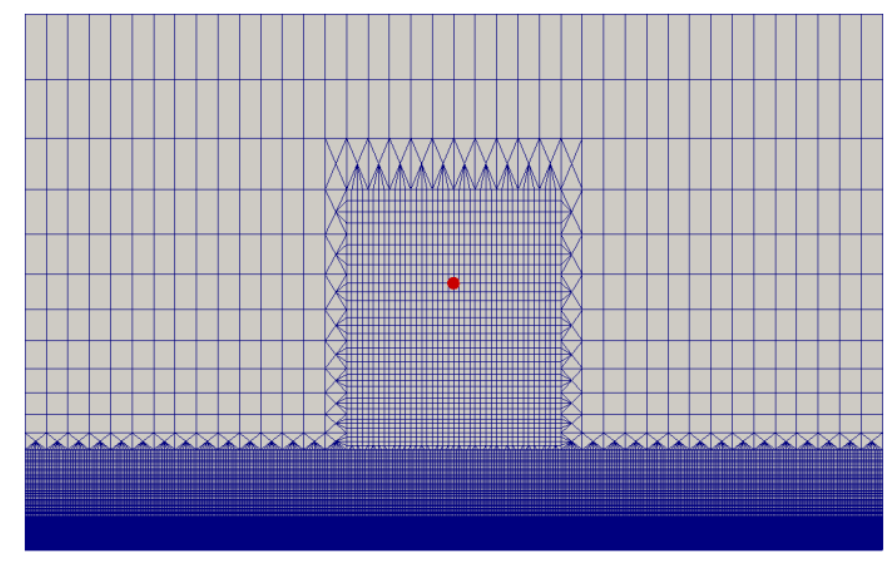

Figure 8: Numerical grid distribution in Case 1.

\section{Discussion}

This section presents the results of the simulations in three cases. Figure 7 presents the temporal variation of droplet shape and vapor formation for Case 1, Case 2, and Case 3. Fig presents the cut sectional view of the domain at $\mathrm{z}=0$ in Case 1 and Case 2. The plane passes through the centers of the droplets. A diagonal plane cutting the centers of droplets is used in Case 3. In Fig. 9, blue color indicates air in the domain, the white color stands for the liquid phase of the fuel and the color with the legend represents the vapor phase of fuel. In vapor phase, red color region indicates $100 \%$ vapor. All cases correspond to film boiling regime where a vapor film can be observed between the droplet and the wall when droplet is in close vicinity of the wall. This film prevents the physical contact between droplet and the wall. This vapor layer formed between the droplets and the wall prevents the droplets from getting into physical contact with the wall. Vapor concentration is high near the part of droplet surface which is exposed to hot wall. Heat flux to the droplet from wall is not by direct physical contact, but by conduction through the vapor film. 
During the simulation, droplets in each case approach the wall with same velocity. Liquid on the surface of the droplet starts to evaporate beyond $1 \mathrm{~ms}$. At $3 \mathrm{~ms}$, droplets reach the hot wall. Vapor film is concentrated between the center region of droplets and the wall. It can be observed that the droplets touch the wall on the circumference but they levitate on the vapor film at the centers. This could be due to more vapor formed at the center compared to the circumference. Since there is no external driving force to enhance vapor diffusion in the surrounding air, mixing is slow and vapor is concentrated near the zone it is formed. Further momentum from the droplet at the center acts against the vapor film. If the Weber number of the droplets is sufficiently low, it doesn't break the film. The momentum changes from vertical to lateral direction resulting in droplet spreading over the vapor film. Droplets continues to spread after impact. In Case 2 and Case 3, droplets merge while spreading and form a single entity. Single droplet in Case 1 and the combined droplet in Case 2 and Case 3 continue to spread in radial direction till a limiting stage. In Case 1, a thin neck region appears at the end of the spreading phase, but a similar neck is not observed in Cases 2 and Cases 3 . This could be because of no interference in spreading of droplet in Case 1, whereas in Case 2 and Case 3, surrounding droplets absorb the momentum while forming the combined droplet entity. This is also reflected in the time and spreading radius. Single droplet spreads for longer duration and forms a disc of larger radius. The combined droplet in Case 2 and Case 3 has much smaller spreading radius. At low Weber numbers, as used in this project, surface tension forces are significant and the droplet doesn't break at the neck region. Instead the droplet tries to regain its spherical shape and retracts. In Case 1, droplet starts to recede after $6 \mathrm{~ms}$ and rebounds from the surface after $9 \mathrm{~ms}$. The reduced spreading in Case 2 and Case 3 results in early onset of receding motion in these cases compared to Case 1. The combined droplet in these cases starts receding much before $5 \mathrm{~ms}$ and starts rebounding at $8 \mathrm{~ms}$. During the rebound phase, droplet appears to oscillate in shape from vertical elongation in the beginning to near spherical shape in the later stages. This is also manifested in terms of oscillations in droplet lift even if the centers of the spherical droplet and elongated droplet doesn't undergo lift in vertical direction. As it can be seen from Case 1 that the droplet from elongated shape at 
$8 \mathrm{~ms}$ transforms to a near spherical shape at $13 \mathrm{~ms}$. Also, the droplet in Case 1 undergoes maximum stretching and hence in receding phase it becomes more elongated shape. In Case 2 and Case 3, droplets undergo minimal spreading and hence do not elongate much in receding phase. Towards the end of rebounding, the droplet weight balances the force exerted by the vapor film as new vapor is continuously generated from the liquid droplet. Overall, it can be observed that the droplet shape and vapor volume fraction distribution of all cases are like those of single droplet case of Nikolopoulos et al [40]. Additionally, from Case 2 and Case 3 of multi-droplets, it can be observed that droplets start to merge together to form a single entity and rebound away from the plate after impact. 


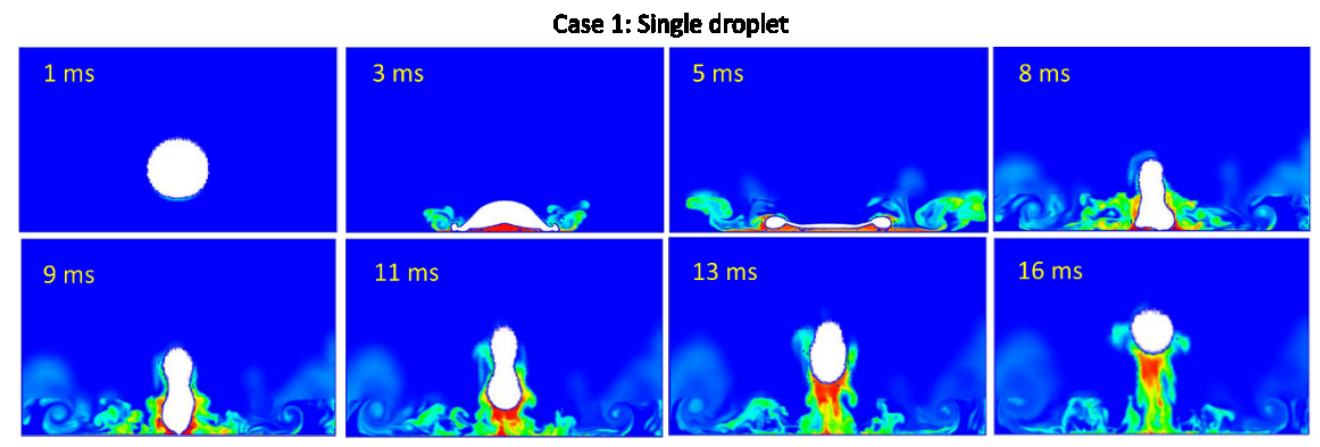

Case 2: 2 droplets
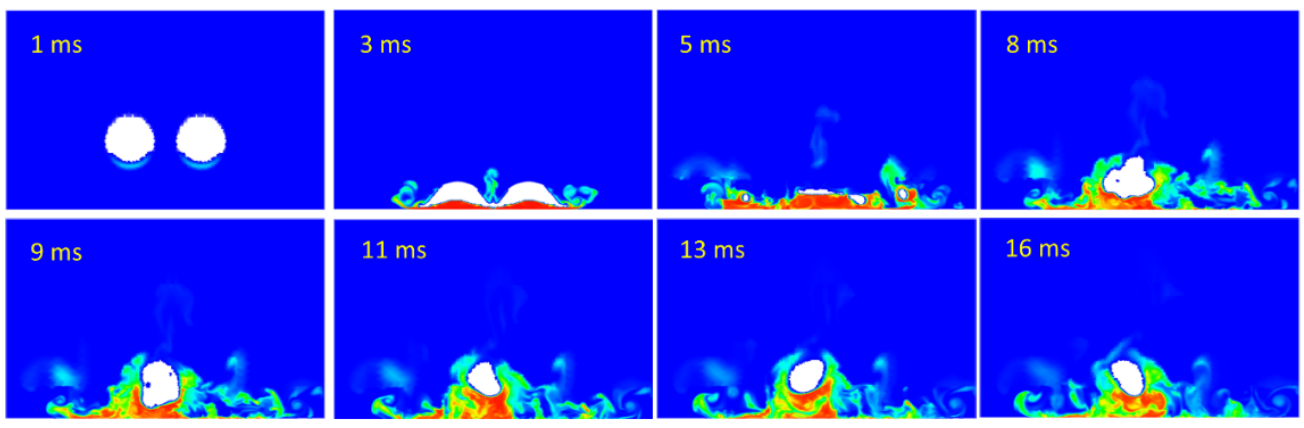

Case 3: 4 droplets
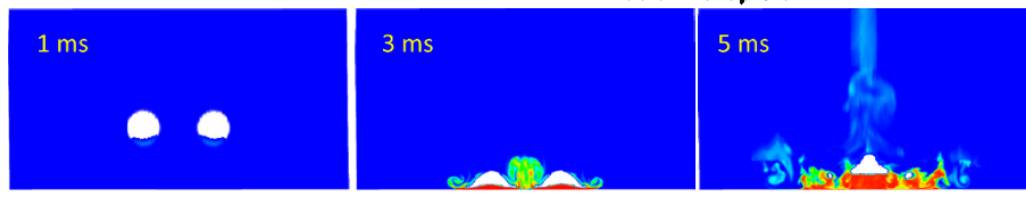

$8 \mathrm{~ms}$

$9 \mathrm{~ms}$
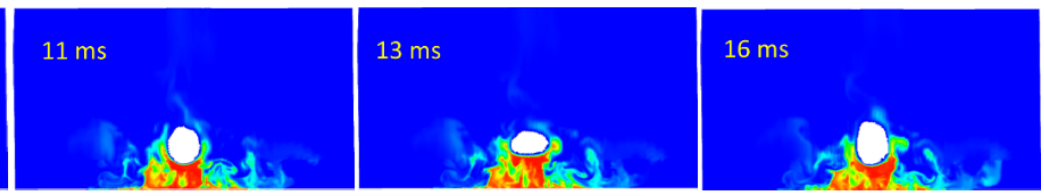

\section{alpha vapor}

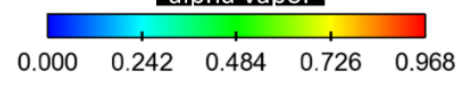

Figure 9: Temporal evolution of liquid and vapor volume fractions.

\section{Droplet Levitation}

In the current study, all the cases are subjected to film boiling, where vapor film between droplet and the wall prevents the droplet from making physical contact with the wall and lifts the droplet above the wall. Droplet levitation is not only a resultant parameter of evaporation., but also an influencing parameter. The amount of the droplet lift influences the surface temperature, vapor distribution around the droplet and hence the rate of evaporation. The minimum of the vertical distances between the surfaces of the droplets to 
the hot wall is considered as droplet levitation. Fig. 10 shows the droplet levitation plotted over time. The droplet diameter in each case is different, but the centers of the droplets are at same vertical distance from the wall. So, initial droplet lift is different in each case. During the simulation, droplets approach wall with some initial velocity and hence droplet lift steadily decreases over time. At $2.5 \mathrm{~ms}$, droplet lift is a small value closer to zero. As droplets spread and continue to evaporate, vapor below the droplet distributes and hence droplet lift increases slightly. When the droplet is spread out to its maximum extent, droplet lift drops again as it tries to balance the force exerted by the vapor. The droplet lift oscillates till a steady state is established between the vapor mass below the droplet and the droplet mass. During the receding motion of the droplet, its lift increases to some extent initially and then decreases as steady state is established. When the droplets rebound from the wall, the lift in Case 1 (single droplet) is much higher compared to that in multiple droplets as the droplet shape change from vertically elongated to spherical between $9 \mathrm{~ms}$ to close to $13 \mathrm{~ms}$ results in more droplet lift. Also the evaporation of liquid mass from the bottom of the droplet results in change in the center of the remaining portion of the droplet. The increase in height of center of droplet results in increased potential energy. This additional potential energy results in droplet lift to be higher than its initial position. In Case 2 and Case 3, stretching is minimal and hence the increase in droplet lift is gradual. Between Case 2 and Case 3, droplet lift was higher in Case 3 between $8 \mathrm{~ms}-12 \mathrm{~ms}$, as droplet recede and rebound motion started early in Case 3. Droplets are arranged in two rows in Case 3 and hence each droplet has two neighboring droplets opposing its spread. Hence droplet in Case 3 starts rising earlier. The droplet levitation becomes almost the same in Case 2 and Case 3 after 14 ms. It can be summarized that droplet spread played an important role in droplet levitation. 


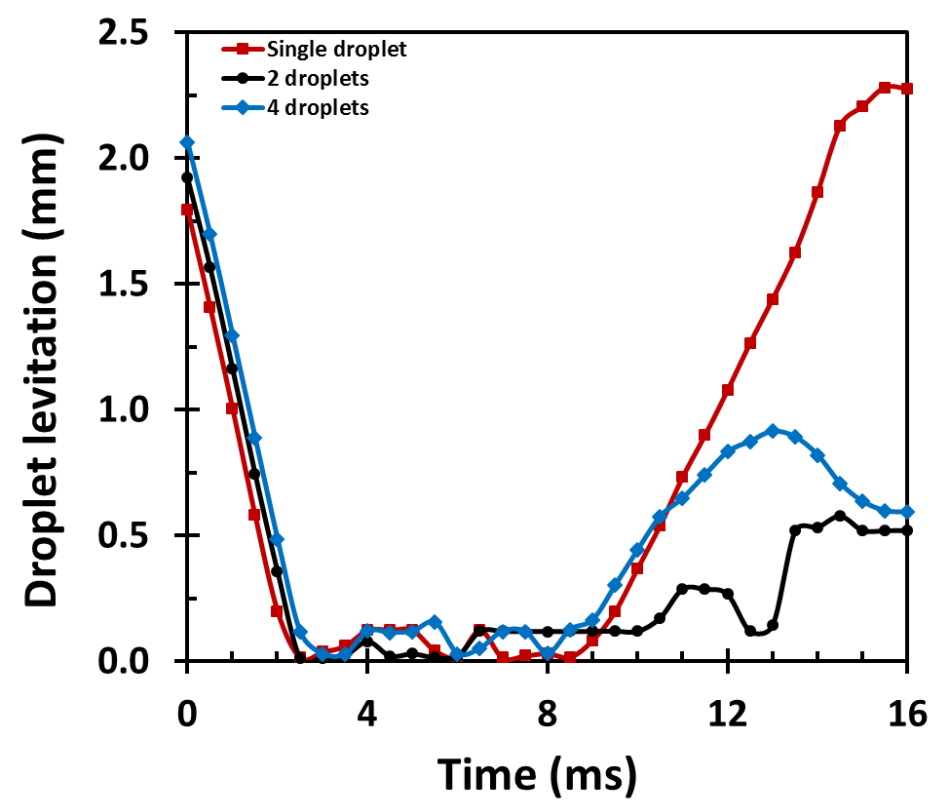

Figure 10: Droplet lift-off height from the wall.

\section{Average Surface Temperature}

The average surface temperature of a droplet is an important parameter associated to phase change of droplet. In this study, surface superheat is not considered. Theoretically, the average surface temperature of droplets with infinitesimally thin surface, undergoing phase change must be saturation temperature. The saturation temperature of n-heptane is $371 \mathrm{~K}$ at atmospheric conditions and hence the droplet undergoes evaporation when the droplet surface reaches that temperature. Irrespective of grid resolution, VOF simulations result in an interface smeared across few cells with finite thickness. Hence, average surface temperature may differ from saturation temperature even with droplet undergoing phase change. Figure 11 presents the results of average surface temperature in all the cases calculated using Equation (4.1).

$$
T_{s, a v g}=\frac{\int T d s}{\int d s}
$$

Droplets in each case are at an initial temperature of 298 K. During simulation, the surface temperature of droplets starts to increase due to continued exposure and movement of droplets towards wall at higher temperature. This leads to transfer of heat to surface of 
droplet and hence surface temperature increases steadily to saturation temperature at about $2.5 \mathrm{~ms}$. Once the surface of droplets reaches saturation temperature, liquid from surface of droplet begins to evaporate. The surface temperature will not increase beyond saturation temperature during the process of evaporation of liquid. It begins to drop during the rebound phase of the droplet as droplet moves away from heated wall to colder domain. The temperature gradient between droplet and the colder domain ensures heat is transferred from droplet to surrounding air. The temperature of the surface drops considerably after 8 ms and the decrease varies between cases. Droplet lift is higher in Case 1 (single droplet), and so the temperature is considerably lower. The decrease in surface temperature is less pronounced in Case 2 ( 2 droplets) and Case 3 (4 droplets). The surface temperature in Case 2 is slightly higher than that in Case 3 beyond $7.5 \mathrm{~ms}$ which is due to less lift occurred in Case 2 compared with that in Case 3 as shown in Fig. 9 and Fig. 10

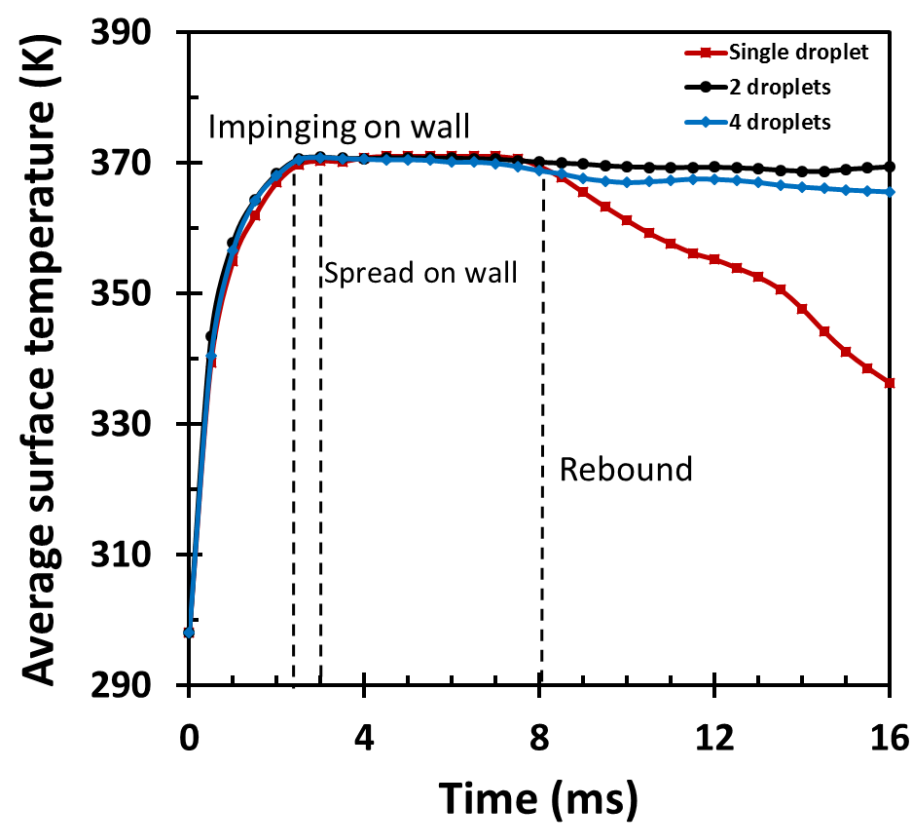

Figure 11: Temporal variation of average surface temperatur 


\section{Liquid mass fraction}

At the beginning of the simulation, all the cases have equal liquid mass though the number of droplets are different. In Figure 12, the liquid mass fraction inside the domain is plotted over time as a percentage of initial liquid mass fraction. Hence, liquid mass fraction is at $100 \%$ at the beginning of the simulation. Droplets are at room temperature initially and hence the liquid mass fraction decreases only slightly at the beginning of the simulation. This transfer of mass is due to mass diffusion from droplet surface to the surrounding air. From Fig, it can be observed that the surface temperature of the droplets steadily increases to saturation temperature. At this point of time, evaporation begins as the vapor pressure of the liquid reaches the surrounding air pressure. As the liquid mass evaporates, there is a sudden decrease in liquid mass fraction and a corresponding increase in vapor mass fraction. Between $2.5 \mathrm{~ms}$ to $4.5 \mathrm{~ms}$, the evaporation rate in Case 3 (4 droplets) is the highest, then Case 2 ( 2 droplets), finally Case 1 (single droplet). The higher evaporation rate in Case 3 is due to larger surface area compared to that in Case 2 and Case 1. For same temperature gradient, the case with larger surface area results in higher cumulative heat transfer and hence higher evaporation rate. Beyond $4.5 \mathrm{~ms}$, droplets in Case 2 and Case 3 merge and hence their surface area decreases. This results in reduction in their evaporation rate. Also, the vapor film between the droplet and the hot wall reduces the heat flux from wall to the droplet. This plays a much bigger role in reducing the evaporation rate in all three cases. In Case 1, droplet spreads and recedes between $4.5 \mathrm{~ms}$ to $8 \mathrm{~ms}$ and continues to evaporate. The higher droplet spread and longer time before droplet begins rebound

phase, results in much lesser liquid mass fraction in Case 1. Case 2 and Case 3 spread a minimal extent and rebound much before $8 \mathrm{~ms}$. This leads to much lesser evaporation rate in Case 2 and Case 3. Higher droplet levitation and less spread lead to less evaporation and higher liquid mass fraction in Case 3 compared to Case 2. Beyond $9 \mathrm{~ms}$, a very high droplet levitation in Case 1 ensures that evaporation is negligible and liquid mass fraction doesn't change considerably. In Case 2, lower droplet levitation compared to that in Case 3 implies that a low evaporation rate will sustain and results in decrease in liquid mass fraction in 
Cases 2. This leads to liquid mass fraction becoming almost equal in Cases 1 and Cases 2 after $15 \mathrm{~ms}$.

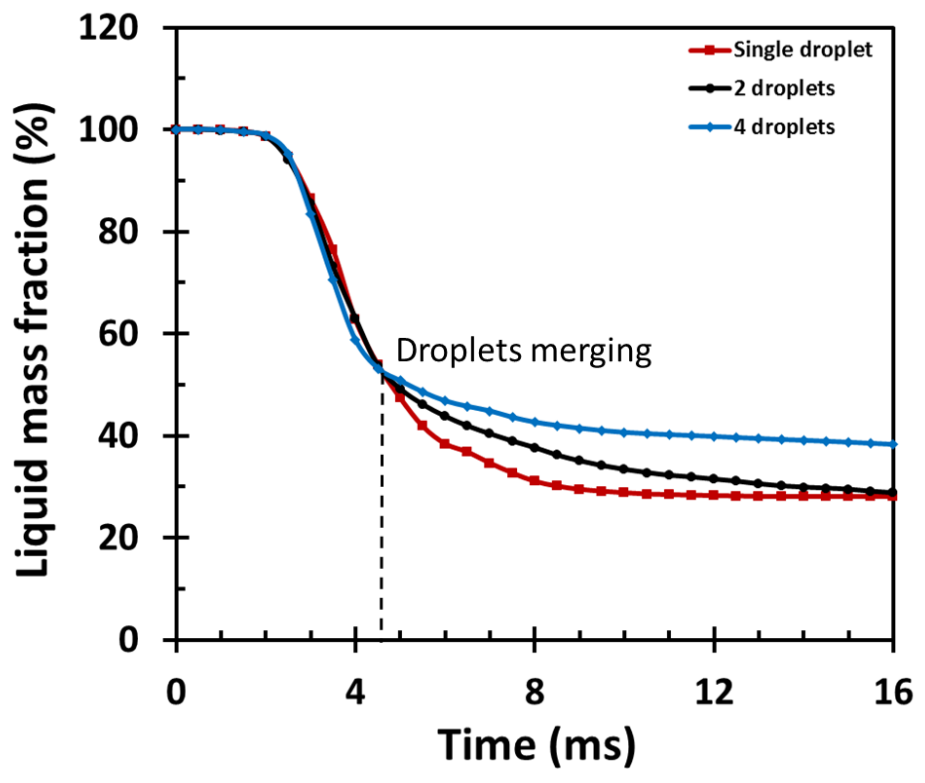

Figure 12: Time dependency of liquid mass fraction. 


\section{CHAPTER 5 CONCLUSIONS AND FUTURE WORK}

This thesis presented an implementation of an evaporation sub-model into an existing solver of VOF method in OpenFOAM CFD code. Existing VOF model and the new mathematical model implemented were validated with the published results. The evaporation solver was used to study the evaporation of multiple spherical droplets impinging on a hot wall. The interaction effects on rate of evaporation in film boiling regime due to spatial distribution of multiple droplets was studied. Droplet levitation, average surface temperature and evaporation rate were compared for three cases including Case 1 (single droplet), Case 2 ( 2 droplets) and Case 3 (4 droplets). The current study leads to few conclusions as summarized below.

* The capability of VOF model implemented in OpenFOAM to simulate binary droplet collisions was successfully demonstrated.

* The simulations successfully predict the droplet levitation characteristic of evaporation above Leidenfrost point.

* In cases with multiple droplets in close proximity, droplet spread is restricted and take less time to recede. This affected both levitation and evaporation rate.

* Droplet levitation reduces as droplet number increases. This reduction is more pronounced if droplets are spread in only one direction. Additionally, if droplets are spread in two directions, reduction in droplet spread leads to increase in lift-off.

* Droplet average surface temperature rapidly increases to saturation temperature and is directly dependent on its proximity with the heated wall.

* Droplet evaporation rate is directly related to surface area as temperature gradients are similar in all three cases. It is higher in Case 3 initially, as it has larger surface area compared to Case 2 and Case 1. When droplets merge together after impact, the spread and surface area are smaller in multi-droplets cases and hence the evaporation rate decreases. 
In future, it is proposed to improve the evaporation solver by implementing piece wiselinear interface calculation (PLIC) interface reconstruction to improve the accuracy of interface representation. The current solver with VOF model is highly dependent on mesh resolution and an accurate description of interface requires high computational resources. To reduce the dependency on mesh, it is proposed to introduce Coupled Level Set Volume of Fluid (CLSVOF) method, which inherits the best of features from Level Set and VOF methods. Additional simulations are planned to evaluate the effect of multiple droplet impingement in more detail in different boiling regimes. Effects of horizontal and vertical distance between droplets, droplet diameter and wall temperature will be explored. Finally, non-spherical shapes will be explored to study evaporation characteristics 


\section{REFERENCES}

[1] O. Reynolds, On the floating of drops on the surface of water depending only on the purity of the surface, Proc. Manchester Lit. Phil. Soc 21(1) (1881).

[2] A. Yarin, Drop impact dynamics: splashing, spreading, receding, bouncing..., Annu. Rev. Fluid Mech. 38 (2006) 159-192.

[3] T.B. Low, R. List, Collision, coalescence and breakup of raindrops. Part I: Experimentally established coalescence efficiencies and fragment size distributions in breakup, Journal of the Atmospheric Sciences 39(7) (1982) 1591-1606.

[4] M. Rein, Phenomena of liquid drop impact on solid and liquid surfaces, Fluid Dynamics Research 12(2) (1993) 61-93.

[5] J. Qian, C. Law, Regimes of coalescence and separation in droplet collision, Journal of Fluid Mechanics 331 (1997) 59-80.

[6] S. Chandra, C. Avedisian, On the collision of a droplet with a solid surface, Proceedings of the Royal Society of London A: Mathematical, Physical and Engineering Sciences, The Royal Society, 1991, pp. 13-41.

[7] C. Bai, A. Gosman, Development of methodology for spray impingement simulation, SAE Technical Paper, 1995.

[8] J. Yang, L. Chow, M. Pais, Nucleate boiling heat transfer in spray cooling, Journal of Heat Transfer 118(3) (1996) 668-671.

[9] S. Kim, D.J. Lee, C.S. Lee, Modeling of binary droplet collisions for application to inter-impingement sprays, International Journal of Multiphase Flow 35(6) (2009) 533-549. [10] J.E. Welch, F.H. Harlow, J.P. Shannon, B.J. Daly, The MAC method-a computing technique for solving viscous, incompressible, transient fluid-flow problems involving free surfaces, Los Alamos Scientific Lab., Univ. of California, N. Mex., 1965.

[11] C.W. Hirt, B.D. Nichols, Volume of fluid (VOF) method for the dynamics of free boundaries, Journal of computational physics 39(1) (1981) 201-225.

[12] S.W. Welch, J. Wilson, A volume of fluid based method for fluid flows with phase change, Journal of computational physics 160(2) (2000) 662-682.

[13] S. Hardt, F. Wondra, Evaporation model for interfacial flows based on a continuumfield representation of the source terms, Journal of Computational Physics 227(11) (2008) 5871-5895.

[14] H. Zhang, Evaporation of a suspended droplet in forced convective high-pressure environments, Combustion Science and Technology 175(12) (2003) 2237-2268.

[15] H. Nomura, Y. Ujiie, H.J. Rath, J.i. Sato, M. Kono, Experimental study on highpressure droplet evaporation using microgravity conditions, Symposium (International) on Combustion, Elsevier, 1996, pp. 1267-1273.

[16] J. Schlottke, B. Weigand, Direct numerical simulation of evaporating droplets, Journal of Computational Physics 227(10) (2008) 5215-5237. 
[17] C. Kunkelmann, P. Stephan, CFD simulation of boiling flows using the volume-offluid method within OpenFOAM, Numerical Heat Transfer, Part A: Applications 56(8) (2009) 631-646.

[18] D. Sun, J. Xu, Q. Chen, Modeling of the evaporation and condensation phase-change problems with FLUENT, Numerical Heat Transfer, Part B: Fundamentals 66(4) (2014) 326-342.

[19] P. O'Rourke, F. Bracco, Modeling of drop interactions in thick sprays and a comparison with experiments, Proceedings of the Institution of Mechanical Engineers 9 (1980) 101-106.

[20] N. Ashgriz, P. Givi, Binary collision dynamics of fuel droplets, International journal of heat and fluid flow 8(3) (1987) 205-210.

[21] N. Ashgriz, J. Poo, Coalescence and separation in binary collisions of liquid drops, Journal of Fluid Mechanics 221 (1990) 183-204.

[22] Y. Jiang, A. Umemura, C. Law, An experimental investigation on the collision behaviour of hydrocarbon droplets, Journal of Fluid Mechanics 234 (1992) 171-190.

[23] M. Rieber, A. Frohn, Three-dimensional Navier-Stokes simulation of binary collisions between droplets of equal size, Journal of Aerosol Science 26 (1995) S929-S930.

[24] M. Nobari, G. Tryggvason, Numerical simulations of three-dimensional drop collisions, AIAA journal 34(4) (1996) 750-755.

[25] Y. Pan, K. Suga, Numerical simulation of binary liquid droplet collision, Physics of Fluids 17(8) (2005) 082105.

[26] N. Nikolopoulos, K.-S. Nikas, G. Bergeles, A numerical investigation of central binary collision of droplets, Computers \& Fluids 38(6) (2009) 1191-1202.

[27] X.G. Li, U. Fritsching, Numerical investigation of binary droplet collisions in all relevant collision regimes, The Journal of Computational Multiphase Flows 3(4) (2011) 207-224.

[28] M. Saroka, N. Ashgriz, M. Movassat, Numerical investigation of head-on binary drop collisions in a dynamically inert environment, Journal of Applied Fluid Mechanics 5(1) (2012) 23-37.

[29] F.H. Harlow, J.P. Shannon, The splash of a liquid drop, Journal of Applied Physics 38(10) (1967) 3855-3866.

[30] G.B. Foote, A numerical method for studying liquid drop behavior: simple oscillation, Journal of Computational Physics 11(4) (1973) 507-530.

[31] J. Madejski, Solidification of droplets on a cold surface, International Journal of Heat and Mass Transfer 19(9) (1976) 1009-1013.

[32] G. Trapaga, J. Szekely, Mathematical modeling of the isothermal impingement of liquid droplets in spraying processes, Metallurgical and Materials Transactions B 22(6) (1991) 901-914.

[33] J. Fukai, Z. Zhao, D. Poulikakos, C.M. Megaridis, O. Miyatake, Modeling of the deformation of a liquid droplet impinging upon a flat surface, Physics of Fluids A: Fluid Dynamics 5(11) (1993) 2588-2599.

[34] M. Bussmann, J. Mostaghimi, S. Chandra, On a three-dimensional volume tracking model of droplet impact, Physics of Fluids 11(6) (1999) 1406-1417. 
[35] S. Kamnis, S. Gu, Numerical modelling of droplet impingement, Journal of Physics D: Applied Physics 38(19) (2005) 3664.

[36] N. Nikolopoulos, A. Theodorakakos, G. Bergeles, Three-dimensional numerical investigation of a droplet impinging normally onto a wall film, Journal of computational physics 225(1) (2007) 322-341.

[37] T. Nguyen, C. Avedisian, Numerical solution for film evaporation of a spherical liquid droplet on an isothermal and adiabatic surface, International journal of heat and mass transfer 30(7) (1987) 1497-1509.

[38] M. Pasandideh-Fard, R. Bhola, S. Chandra, J. Mostaghimi, Deposition of tin droplets on a steel plate: simulations and experiments, International Journal of Heat and Mass Transfer 41(19) (1998) 2929-2945.

[39] D.J. Harvie, D.F. Fletcher, A hydrodynamic and thermodynamic simulation of droplet impacts on hot surfaces, Part I: theoretical model, International Journal of Heat and Mass Transfer 44(14) (2001) 2633-2642.

[40] N. Nikolopoulos, A. Theodorakakos, G. Bergeles, A numerical investigation of the evaporation process of a liquid droplet impinging onto a hot substrate, International Journal of Heat and Mass Transfer 50(1) (2007) 303-319.

[41] A.V. Mahulkar, G.B. Marin, G.J. Heynderickx, Droplet-wall interaction upon impingement of heavy hydrocarbon droplets on a heated wall, Chemical Engineering Science 130 (2015) 275-289.

[42] G. Soriano, J.L. Alvarado, Y.P. Lin, Experimental characterization of single and multiple droplet impingement on surfaces subject to constant heat flux conditions, 2010 14th International Heat Transfer Conference, American Society of Mechanical Engineers, 2010, pp. 707-715.

[43] S. Lewis, L. Anumolu, M. Trujillo, Numerical simulations of droplet train and free surface jet impingement, International Journal of Heat and Fluid Flow 44 (2013) 610-623. [44] H.G. Weller, G. Tabor, H. Jasak, C. Fureby, A tensorial approach to computational continuum mechanics using object-oriented techniques, Computers in physics 12(6) (1998) 620-631.

[45] H. Jasak, Error analysis and estimation for finite volume method with applications to fluid flow, (1996).

[46] J. Brackbill, D.B. Kothe, C. Zemach, A continuum method for modeling surface tension, Journal of computational physics 100(2) (1992) 335-354.

[47] P. Keller, P. Nikrityuk, B. Meyer, M. Müller-Hagedorn, Numerical simulation of evaporating droplets with chemical reactions using a volume of fluid method, 7th International Conference on Multiphase Flows, 2010. 


\section{APPENDIX}

\section{Chapter 4}

$8 / 2 / 2017$

Michigan Technological University Mail - Permission to use material from my SAE paper in my thesis

Michigan Tech

Sathya Potham <spotham@mtu.edu>

\section{Permission to use material from my SAE paper in my thesis}

Mandy May <Mandy.May@sae.org>

To: Sathya Potham <spotham@mtu.edu>

Sun, Jul 30, 2017 at 2:35 PM

Thank you for contacting SAE International, Sathya.

Permission is granted for your use of your original content in your dissertation as outlined in your email below.

Please consider the following conditions:

-Permission is granted for non-exclusive English language rights, and for the specific use as indicated in your email;

-A new Copyright Permission Request is required for any further use of this material, other than that specified within your original Request;

-The SAE material must be clearly identified and include the following statement "Reprinted with permission Copyright $@ x x x x$ SAE International. Further distribution of this material is not permitted without prior permission from SAE."?

-We also request that you include a complete reference to the SAE document in the reference section for each figure used;

-This permission does not cover any third-party copyrighted work which may appear in the material requested;

-Any language use, other than specified within your email, requires a new copyright request and additional fee;

-Licensor's use of this material, in whole or in part, is entirely its responsibility, and SAE International does not warrant or is not responsible for any use of the material.

Best wishes on your dissertation,

Mandy

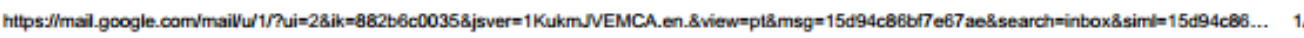




\section{Permission to use material from my SAE paper in my thesis}

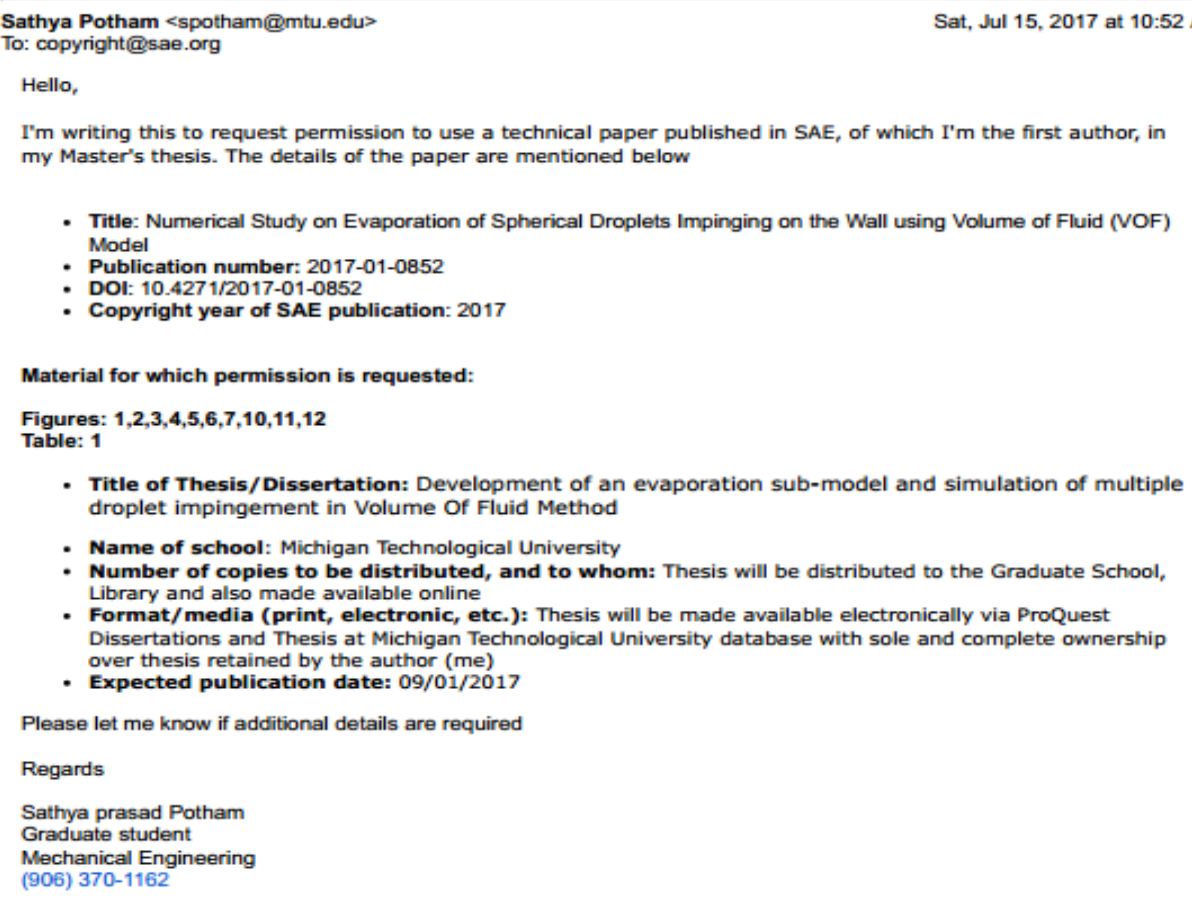

- Title of Thesis/Dissertation: Development of an evaporation sub-model and simulation of multiple droplet impingement in Volume Of Fluid Method

- Name of school: Michigan Technological University

- Number of copies to be distributed, and to whom: Thesis will be distributed to the Graduate School, Library and also made available online

- Format/media (print, electronic, etc.): Thesis will be made available electronically via ProQuest Dissertations and Thesis at Michigan Technological University database with sole and complete ownership over thesis retained by the author (me)

- Expected publication date: 09/01/2017

Please let me know if additional details are required

Regards

Sathya prasad Potham

Graduate student

Mechanical Engineering

(906) $370-1162$ 


\section{Figures 1 and 2}

8/2/2017

Michigan Technological University Mal - FW: Permission Request

Michigan Tech

Sathya Potham <spotham@mtu.edu>

\section{FW: Permission Request}

Georgia Stratton <gstratton@cambridge.org>

Tue, Aug 1, 2017 at 9:28 AM

Georgia Stratton <gstratton@cambridge.org>
To: "spotham@mtu.edu" <spotham@mtu.edu>

Tu, Aug 1, 2017 at 9:28 Ad

Dear Sathya prasad Potham,

Thank you for your request to reproduce two figures from the below title in your forthcoming work provisionally entitled Development of an Evaporation sub-model and simulation of multiple droplet impingement in Volume of Fluid method. Cambridge University Press are pleased to grant non-exclusive permission, free of charge, for this specific one time use, on the understanding you have checked that we do not acknowledge any other source for the figures. This permission material must be sought by you from the copyright owner concerned.

Please ensure full acknowledgement appears in your work.

Further information can be found on our website at the following link:

http://www.cambridge.org/about-us/rights-permissions/permissions/

Yours sincerely,

Georgia Stratton,

Permissions Sales Administrator | Permissions Sales | Academic Books \& Journals, ELT \& Education

Cambridge University Press

University Printing House | Shaftesbury Road | Cambridge | CB2 8BS, UK

\section{CAMBRIDGE}

OUR PERMISSIONS LICENSING

Chex Here for more detals $>>$

-.-Original Message-...

From: Permissions request [mailto:noreply@cambridge.org]

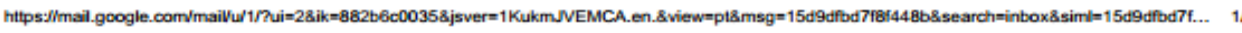


Figure 3

\begin{tabular}{|c|c|}
\hline \multirow[t]{2}{*}{$8 / 2 / 2017$} & \multirow[t]{2}{*}{ RightsLink Printable License } \\
\hline & \\
\hline \multicolumn{2}{|c|}{$\begin{array}{l}\text { This Agreement between Mr. Sathya prasad Potham ("You") and Elsevier ("Elsevier") } \\
\text { consists of your license details and the terms and conditions provided by Elsevier and } \\
\text { Copyright Clearance Center. }\end{array}$} \\
\hline License Number & 4160870819323 \\
\hline License date & Aug 02, 2017 \\
\hline Licensed Content Publisher & Elsevier \\
\hline Licensed Content Publication & Journal of Computational Physics \\
\hline Licensed Content Title & $\begin{array}{l}\text { Three-dimensional numerical investigation of a droplet impinging } \\
\text { normally onto a wall film }\end{array}$ \\
\hline Licensed Content Author & N. Nikolopoulos,A. Theodorakakos,G. Bergeles \\
\hline Licensed Content Date & Jul 1, 2007 \\
\hline Licensed Content Volume & 225 \\
\hline Licensed Content Issue & 1 \\
\hline Licensed Content Pages & 20 \\
\hline Start Page & 322 \\
\hline End Page & 341 \\
\hline Type of Use & reuse in a thesis/dissertation \\
\hline $\begin{array}{l}\text { Intended publisher of new } \\
\text { work }\end{array}$ & other \\
\hline Portion & figures/tables/illustrations \\
\hline $\begin{array}{l}\text { Number of } \\
\text { figures/tables/illustrations }\end{array}$ & 1 \\
\hline Format & both print and electronic \\
\hline $\begin{array}{l}\text { Are you the author of this } \\
\text { Elsevier article? }\end{array}$ & No \\
\hline Will you be translating? & No \\
\hline Original figure numbers & Figure $8(a)$ \\
\hline $\begin{array}{l}\text { Title of your } \\
\text { thesis/dissertation }\end{array}$ & $\begin{array}{l}\text { DEVELOPMENT OF AN EVAPORATION SUB-MODEL AND SIMULATION } \\
\text { OF MULTIPLE DROPLET IMPINGEMENT IN VOLUME OF FLUID } \\
\text { METHOD }\end{array}$ \\
\hline Expected completion date & Aug 2017 \\
\hline $\begin{array}{l}\text { Estimated size (number of } \\
\text { pages) }\end{array}$ & 60 \\
\hline Requestor Location & $\begin{array}{l}\text { Mr. Sathya prasad Potham } \\
2855 \text { Cherokee Dr Apt } 22\end{array}$ \\
\hline & $\begin{array}{l}\text { WATERFORD, MI } 48328 \\
\text { United States } \\
\text { Attn: Mr. Sathya prasad Potham }\end{array}$ \\
\hline Publisher Tax ID & $98-0397604$ \\
\hline
\end{tabular}




\section{Figures 5 and 6}

